\title{
Magnesiation of Electron-Rich Aryl Bromides and Their Use in Nickel-Catalyzed Cross-Coupling Reactions
}

\author{
Stephen Y. W. Lau, ${ }^{* \dagger}$ Greg Hughes, ${ }^{\dagger}$ Paul D. O’Shea ${ }^{\dagger}$ and Ian W. Davies ${ }^{\ddagger}$ \\ ${ }^{\dagger}$ Department of Process Research, Merck Frosst Centre for Therapeutic Research, 16711 Trans \\ Canada Highway, Kirkland, QC, Canada, H9H 3L1 and 'Department of Process Research, Merck \\ Research Laboratories, P.O. Box 2000, Rahway, NJ, USA, 07065. \\ *stephen_lau@merck.com
}

Supporting Information Table of Contents:

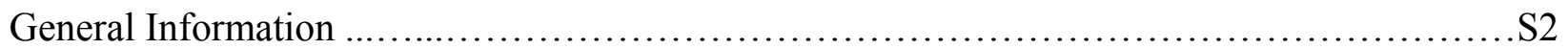

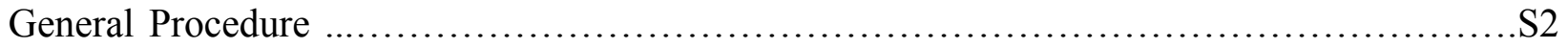

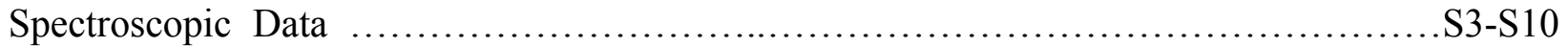

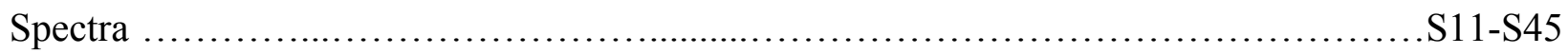




\section{General Information:}

All reactions were carried out under nitrogen in oven-dried glassware. All catalysts and reagents were obtained from commercial sources and were used without further purification. Anhydrous THF was obtained from commercial sources and degassed under $\mathrm{N}_{2}$ prior to use. Yields refer to isolated yields of compounds estimated to be $>95 \%$ pure as determined by ${ }^{1} \mathrm{H}$ NMR or GC analysis. Proton and carbon NMR spectra were obtained on a Bruker Avance $400\left({ }^{1} \mathrm{H} 400\right.$ $\left.\mathrm{MHz},{ }^{13} \mathrm{C} 100 \mathrm{MHz}\right)$ or Bruker Avance $500\left({ }^{1} \mathrm{H} 500 \mathrm{MHz},{ }^{13} \mathrm{C} 126 \mathrm{MHz}\right)$ spectrometer. GC-MS analysis was performed on an Agilent 6890 gas chromatograph with an Agilent 5973 mass selective detector. HRMS analyses were obtained via ESI with $\mathrm{Ag}^{+}$added in the carrier for ionization resulting in $\mathrm{M}+\mathrm{Ag}$ masses.

\section{General procedure:}

In a round-bottomed flask, an aryl or vinyl bromide, chloride, tosylate or triflate ( $5 \mathrm{mmol}, 1$ equiv) was dissolved in $10 \mathrm{~mL}$ THF. $\mathrm{NiCl}_{2} \bullet \mathrm{dppp}(1 \mathrm{~mol} \%)$ was added, cooled to $0{ }^{\circ} \mathrm{C}$ and degassed with $\mathrm{N}_{2}$ for 10 min.

In a separate round-bottomed flask, THF $(7.5 \mathrm{~mL})$ was cooled to $0{ }^{\circ} \mathrm{C} . \mathrm{n}-\mathrm{BuLi}(2 \mathrm{~mL}, 2.5 \mathrm{M}$ in hexanes, $5 \mathrm{mmol}, 1$ equiv) was added followed by $\mathrm{n}-\mathrm{BuMgCl}(1.25 \mathrm{~mL}, 2 \mathrm{M}$ in THF, $2.5 \mathrm{mmol}$, 0.5 equiv) forming a light yellow solution. The solution was stirred at $0{ }^{\circ} \mathrm{C}$ for $5 \mathrm{~min}$. A solution of aryl bromide (6.5 mmol, 1.3 equiv) in THF $(6.5 \mathrm{~mL})$ was added over $2 \mathrm{~min}$, and stirred at $0{ }^{\circ} \mathrm{C}$ for an additional $5 \mathrm{~min}$, whereupon $\mathrm{GC}$ analysis showed the complete disappearance of the starting aryl bromide. The resulting magnesiate solution was added to the cold vinyl bromide/ $\mathrm{NiCl}_{2} \bullet$ dppp suspension dropwise over 10-15 min and the reaction was stirred an additional 15-30 $\mathrm{min}$ at $0{ }^{\circ} \mathrm{C}$. The reaction was diluted with MTBE $(10 \mathrm{~mL})$ and quenched with $\mathrm{HCl}(10 \mathrm{~mL}, 1 \mathrm{M})$. The layers were separated and the organic layer was washed twice with brine $(10 \mathrm{~mL})$, treated with $1 \mathrm{~g}$ activated charcoal (Darco KB or equivalent) to remove nickel, dried with $\mathrm{MgSO}_{4}$, filtered and concentrated. The residue was triturated in a minimal amount of hexanes and chromatographed (hexanes) to give the desired product. All compounds obtained in $>99 \%$ purity by GC analysis. 


\section{1,2-diphenoxyethane (3) ${ }^{1}$}<smiles>c1ccc(OCCOc2ccccc2)cc1</smiles>

${ }^{1} \mathrm{H}$ NMR (500 MHz, $\left.\mathrm{CDCl}_{3}\right): \delta 7.30(\mathrm{t}, \mathrm{J}=7.82 \mathrm{~Hz}, 4 \mathrm{H}), 7.00-6.93(\mathrm{~m}, 6 \mathrm{H}), 4.32(\mathrm{~s}, 4 \mathrm{H})$. Spectral data are consistent with literature data.

\section{1-(Biphen-4-yloxy)-2-phenoxyethane (4)}<smiles>c1ccc(OCCOc2ccc(-c3ccccc3)cc2)cc1</smiles>

${ }^{1} \mathrm{H}$ NMR (500 MHz, $\left.\mathrm{CDCl}_{3}\right) \delta$ 7.57-7.51 (m, $\left.3 \mathrm{H}\right), 7.40(\mathrm{t}, \mathrm{J}=7.62 \mathrm{~Hz}, 2 \mathrm{H}), 7.31-7.25(\mathrm{~m}, 3 \mathrm{H})$, $7.02(\mathrm{~d}, \mathrm{~J}=8.59 \mathrm{~Hz}, 2 \mathrm{H}), 6.98-6.92(\mathrm{~m}, 4 \mathrm{H}), 4.39-4.33(\mathrm{~m}, 4 \mathrm{H}) .{ }^{13} \mathrm{C} \mathrm{NMR}(126 \mathrm{MHz}$, $\left.\mathrm{CDCl}_{3}\right): \delta 158.6,158.2,134.2,129.5,129.4,128.7,128.2,126.7,121.1,121.0,115.0,114.7$, 66.6, 66.4. Mass spectrum, m/z (relative intensity, \%): $290\left(100, \mathrm{M}^{+}\right), 170\left(100, \mathrm{M}^{+}-\right.$ $\left.\mathrm{C}_{6} \mathrm{H}_{5} \mathrm{OC}_{2} \mathrm{H}_{3}\right)$. Exact mass for $\mathrm{C}_{20} \mathrm{H}_{18} \mathrm{O}_{2} \mathrm{Ag}(\mathrm{M}+\mathrm{Ag})$ : calculated 397.0358, found 397.0351.

\section{4-Methoxybiphenyl (7) ${ }^{2}$}<smiles>COc1ccc(-c2ccccc2)cc1</smiles>

\footnotetext{
${ }^{1}$ Brennan, M. P. J., Brettle, R. J. Chem. Soc. Perkin Trans. 1 1973; 257-261.

${ }^{2}$ Hagio, H., Sugiura, M., Kobayashi, S. Org. Lett. 2006, 8, 375.
} 
${ }^{1} \mathrm{H}$ NMR (400 MHz, $\left.\mathrm{CDCl}_{3}\right): \delta$ 7.58-7.49 (4 H, m), $7.41(2 \mathrm{H}, \mathrm{t}, \mathrm{J}=7.55 \mathrm{~Hz}), 7.29(1 \mathrm{H}, \mathrm{t}, \mathrm{J}=$ $7.42 \mathrm{~Hz}), 6.97(2 \mathrm{H}, \mathrm{d}, \mathrm{J}=8.22 \mathrm{~Hz}), 3.84(4 \mathrm{H}, \mathrm{s})$. Spectral data are consistent with literature data.

\section{4,4'-Dimethoxybiphenyl (8) ${ }^{3}$}<smiles>COc1ccc(-c2ccc(OC)cc2)cc1</smiles>

${ }^{1} \mathrm{H}$ NMR (400 MHz, $\left.\mathrm{CDCl}_{3}\right): \delta 7.46(\mathrm{~d}, \mathrm{~J}=8.72 \mathrm{~Hz}, 3 \mathrm{H}), 6.94(\mathrm{~d}, \mathrm{~J}=8.74 \mathrm{~Hz}, 3 \mathrm{H}), 3.83(\mathrm{~s}, 6$ H). Spectral data are consistent with literature data.

\section{4-Methoxybiphenyl (Table 3, Entry 1)}<smiles>COc1ccc(-c2ccccc2)cc1</smiles>

${ }^{1} \mathrm{H}$ NMR (400 MHz, $\left.\mathrm{CDCl}_{3}\right): \delta$ 7.58-7.49 (4 H, m), $7.41(2 \mathrm{H}, \mathrm{t}, \mathrm{J}=7.55 \mathrm{~Hz}), 7.29(1 \mathrm{H}, \mathrm{t}, \mathrm{J}=$ $7.42 \mathrm{~Hz}), 6.97(2 \mathrm{H}, \mathrm{d}, \mathrm{J}=8.22 \mathrm{~Hz}), 3.84(4 \mathrm{H}, \mathrm{s})$. Spectral data are consistent with compound 7.

\section{4-Methoxybiphenyl (Table 3, Entry 2)}<smiles>COc1ccc(-c2ccccc2)cc1</smiles>

${ }^{1} \mathrm{H}$ NMR (400 MHz, $\left.\mathrm{CDCl}_{3}\right): \delta$ 7.58-7.49 (4 H, m), $7.40(2 \mathrm{H}, \mathrm{t}, \mathrm{J}=7.55 \mathrm{~Hz}), 7.28(1 \mathrm{H}, \mathrm{t}, \mathrm{J}=$ $7.42 \mathrm{~Hz}), 6.97(2 \mathrm{H}, \mathrm{d}, \mathrm{J}=8.22 \mathrm{~Hz}), 3.83(4 \mathrm{H}, \mathrm{s})$. Spectral data are consistent with compound 7.

\footnotetext{
${ }^{3}$ Liu, L., Zhang, Y., Xin, B. J. Org. Chem. 2006, 71, 3994.
} 


\section{4-Methoxybiphenyl (Table 3, Entry 3)}<smiles>COc1ccc(-c2ccccc2)cc1</smiles>

${ }^{1} \mathrm{H}$ NMR (400 MHz, $\left.\mathrm{CDCl}_{3}\right): \delta$ 7.58-7.49 (4 H, m), $7.40(2 \mathrm{H}, \mathrm{t}, \mathrm{J}=7.55 \mathrm{~Hz}), 7.29(1 \mathrm{H}, \mathrm{t}, \mathrm{J}=$ $7.42 \mathrm{~Hz}), 6.97(2 \mathrm{H}, \mathrm{d}, \mathrm{J}=8.22 \mathrm{~Hz}), 3.83(4 \mathrm{H}, \mathrm{s})$. Spectral data are consistent with compound 7.

\section{4-Methoxybiphenyl (Table 3, Entry 4)}<smiles>COc1ccc(-c2ccccc2)cc1</smiles>

${ }^{1} \mathrm{H}$ NMR (400 MHz, $\left.\mathrm{CDCl}_{3}\right): \delta$ 7.58-7.49 (4 H, m), $7.40(2 \mathrm{H}, \mathrm{t}, \mathrm{J}=7.55 \mathrm{~Hz}), 7.28(1 \mathrm{H}, \mathrm{t}, \mathrm{J}=$ $7.42 \mathrm{~Hz}), 6.97(2 \mathrm{H}, \mathrm{d}, \mathrm{J}=8.22 \mathrm{~Hz}), 3.83(4 \mathrm{H}, \mathrm{s})$. Spectral data are consistent with compound 7.

\section{1-Methoxy-4-(2-propenyl)benzene (Table 3, Entry 5) ${ }^{4}$}<smiles>C=C(C)c1ccc(OC)cc1</smiles>

${ }^{1} \mathrm{H}$ NMR (400 MHz, $\left.\mathrm{CDCl}_{3}\right): \delta 7.40(\mathrm{~d}, \mathrm{~J}=8.56 \mathrm{~Hz}, 2 \mathrm{H}), 6.85(\mathrm{~d}, \mathrm{~J}=8.65 \mathrm{~Hz}, 2 \mathrm{H}), 5.27(\mathrm{~s}, 1$ H), $4.97(\mathrm{~s}, 1 \mathrm{H}), 3.80(\mathrm{~s}, 3 \mathrm{H}), 2.11(\mathrm{~s}, 3 \mathrm{H})$. Spectral data are consistent with literature data.

\footnotetext{
${ }^{4}$ Comer, E., Organ, M., Hynes, S. J. J. Am. Chem. Soc. 2004, 126, 16087.
} 


\section{1-Cyclohexylidenemethyl-4-methoxybenzene (Table 3, Entry 6) ${ }^{5}$}<smiles>COc1ccc(C=C2CCCCC2)cc1</smiles>

${ }^{1} \mathrm{H}$ NMR $\left(500 \mathrm{MHz}, \mathrm{CDCl}_{3}\right): \delta 7.11(\mathrm{~d}, \mathrm{~J}=8.42 \mathrm{~Hz}, 2 \mathrm{H}) ; 6.83(\mathrm{~d}, \mathrm{~J}=8.52 \mathrm{~Hz}, 2 \mathrm{H}) ; 6.14(\mathrm{~s}, 1$ $\mathrm{H}) ; 3.78(\mathrm{~s}, 3 \mathrm{H}) ; 2.33(\mathrm{t}, \mathrm{J}=5.94 \mathrm{~Hz}, 2 \mathrm{H}) ; 2.21(\mathrm{t}, \mathrm{J}=5.68 \mathrm{~Hz}, 2 \mathrm{H}) ; 1.63-1.54(\mathrm{~m}, 4 \mathrm{H})$; 1.56-1.50 (m, $2 \mathrm{H})$. Spectral data are consistent with literature data.

1-Methoxy-4-(2-methylpropenyl)benzene (Table 3, Entry 7)<smiles>COc1ccc(C=C(C)C)cc1</smiles>

${ }^{1} \mathrm{H}$ NMR (400 MHz, $\left.\mathrm{CDCl}_{3}\right): \delta 7.14(\mathrm{~d}, \mathrm{~J}=8.36 \mathrm{~Hz}, 2 \mathrm{H}), 6.84(\mathrm{~d}, \mathrm{~J}=8.39 \mathrm{~Hz}, 2 \mathrm{H}), 6.19$ (s, 1 H), $3.79(\mathrm{~s}, 3 \mathrm{H}), 1.87(\mathrm{~s}, 3 \mathrm{H}), 1.83(\mathrm{~s}, 3 \mathrm{H})$. Spectral data are consistent with literature data.

\section{1-Methoxy-4-(2-methylpropenyl)benzene (Table 3, Entry 8)}<smiles>COc1ccc(C=C(C)C)cc1</smiles>

${ }^{1} \mathrm{H}$ NMR (400 MHz, $\left.\mathrm{CDCl}_{3}\right): \delta 7.14(\mathrm{~d}, \mathrm{~J}=8.38 \mathrm{~Hz}, 2 \mathrm{H}), 6.84(\mathrm{~d}, \mathrm{~J}=8.38 \mathrm{~Hz}, 2 \mathrm{H}), 6.20$ (s, 1 H), $3.79(\mathrm{~s}, 3 \mathrm{H}), 1.87(\mathrm{~s}, 3 \mathrm{H}), 1.83(\mathrm{~s}, 3 \mathrm{H})$. Spectral data are consistent with Table 3, Entry 7.

${ }^{5}$ Satoh, T., Hanaki, N., Yamada, N., Asano, T. Tetrahedron 2000, 56, 6223.

${ }^{6}$ Happer, D. A. R.; Steenson, B. E. J. Chem. Soc. Perkin Trans. 2 1988, 19. 


\section{2-(4-Methoxyphenyl)pyridine (Table 3, Entry 9) ${ }^{7}$}<smiles>COc1ccc(-c2ccccn2)cc1</smiles>

${ }^{1} \mathrm{H}$ NMR (400 MHz, $\left.\mathrm{CDCl}_{3}\right): \delta 8.63(\mathrm{~d}, \mathrm{~J}=4.70 \mathrm{~Hz}, 1 \mathrm{H}), 7.93(\mathrm{~d}, \mathrm{~J}=8.32 \mathrm{~Hz}, 2 \mathrm{H})$, 7.73-7.61 $(\mathrm{m}, 2 \mathrm{H}), 7.15(\mathrm{dd}, \mathrm{J}=5.95 \mathrm{~Hz}, 1 \mathrm{H}), 6.98(\mathrm{~d}, \mathrm{~J}=8.40 \mathrm{~Hz}, 2 \mathrm{H}), 3.84(\mathrm{~s}, 3 \mathrm{H})$. Spectral data are consistent with literature data.

\section{4-methoxy-4'-trifluoromethylbiphenyl (Table 3, Entry 10) ${ }^{8}$}<smiles>COc1ccc(-c2ccc(C(F)(F)F)cc2)cc1</smiles>

${ }^{1} \mathrm{H}$ NMR (400 MHz, $\left.\mathrm{CDCl}_{3}\right): \delta 7.64(\mathrm{~s}, 4 \mathrm{H}), 7.53(\mathrm{~d}, \mathrm{~J}=8.82 \mathrm{~Hz}, 2 \mathrm{H}), 6.99(\mathrm{~d}, \mathrm{~J}=8.82 \mathrm{~Hz}, 2$ $\mathrm{H}), 3.84(\mathrm{~s}, 3 \mathrm{H})$. Spectral data are consistent with literature data.

\section{3,5-Dimethoxybiphenyl (Table 4, Entry 1) ${ }^{9}$}<smiles>COc1cc(OC)cc(-c2ccccc2)c1</smiles>

\footnotetext{
${ }^{7}$ Parmentier, M., Gros, P., Fort, Y. Tetrahedron 2005, 61, 3261.

${ }^{8}$ Molander, G. A.; Petrillo, D. E.; Landzberg, N. R.; Rohanna, J. C.; Biolatto, B. Syn. Lett. 2005, 11, 1763.

${ }^{9}$ Song, C., Ma, Y., Chai, Q., Ma, C., Jiang, W., Andrus, M. B. Tetrahedron 2005, 61, 7438.
} 
${ }^{1} \mathrm{H}$ NMR (400 MHz, $\mathrm{CDCl}_{3}$ ): $\delta 7.57$ (d, J = 7.64 Hz, $\left.2 \mathrm{H}\right), 7.42(\mathrm{~m}, 2 \mathrm{H}), 7.34(\mathrm{~m}, 1 \mathrm{H}), 6.73$ (d, $\mathrm{J}=2.27 \mathrm{~Hz}, 2 \mathrm{H}), 6.47(\mathrm{t}, \mathrm{J}=2.27 \mathrm{~Hz}, 1 \mathrm{H}), 3.84(\mathrm{~s}, 6 \mathrm{H})$. Spectral data are consistent with literature data.

\section{1,3-dimethoxy-5-(2-propenyl)benzene (Table 4, Entry 2)}<smiles>C=C(C)c1cc(OC)cc(OC)c1</smiles>

${ }^{1} \mathrm{H}$ NMR $\left(500 \mathrm{MHz}, \mathrm{CDCl}_{3}\right): \delta 6.60(\mathrm{~d}, \mathrm{~J}=2.28 \mathrm{~Hz}, 2 \mathrm{H}), 6.38(\mathrm{t}, \mathrm{J}=2.28 \mathrm{~Hz}, 1 \mathrm{H}), 5.33(\mathrm{~s}, 1$ $\mathrm{H})$, 5.09-5.03 (m, $1 \mathrm{H}), 3.79(\mathrm{~s}, 6 \mathrm{H}), 2.11(\mathrm{~s}, 3 \mathrm{H}) .{ }^{13} \mathrm{C} \mathrm{NMR}\left(125 \mathrm{MHz}, \mathrm{CDCl}_{3}\right): \delta 160.6$, 143.5, 143.3, 112.8, 104.0, 99.2, 55.3, 21.9. Mass spectrum, m/z (relative intensity, \%): 178 $\left(\mathrm{M}^{+}, 100\right), 163\left(\mathrm{M}^{+}-\mathrm{CH}_{3}, 14\right)$. Exact mass for $\mathrm{C}_{11} \mathrm{H}_{14} \mathrm{O}_{2} \mathrm{Ag}(\mathrm{M}+\mathrm{Ag})$ : calculated 285.0045, found 285.0049 .

\section{1,3-Dimethyoxy-5-(2-methylpropenyl)benzene (Table 4, Entry 3)}

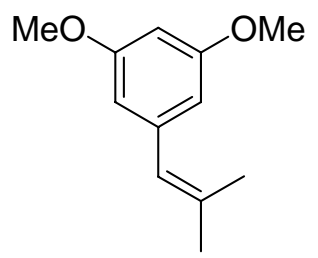

${ }^{1} \mathrm{H}$ NMR (400 MHz, $\left.\mathrm{CDCl}_{3}\right): \delta 6.37(\mathrm{~d}, \mathrm{~J}=2.29 \mathrm{~Hz}, 2 \mathrm{H}), 6.31(\mathrm{t}, \mathrm{J}=2.29 \mathrm{~Hz}, 1 \mathrm{H}), 6.20(\mathrm{~s}, 1$ $\mathrm{H}), 3.77(\mathrm{~s}, 7 \mathrm{H}), 1.88(\mathrm{~s}, 3 \mathrm{H}), 1.86(\mathrm{~s}, 3 \mathrm{H}) .{ }^{13} \mathrm{C} \mathrm{NMR}\left(100 \mathrm{MHz}, \mathrm{CDCl}_{3}\right): \delta$ 160.4, 140.6, 136.0, 125.1, 106.8, 98.1, 55.2, 26.8, 19.5. Mass spectrum, m/z (relative intensity, \%): $192\left(\mathrm{M}^{+}\right.$, 100), $177\left(\mathrm{M}^{+}-\mathrm{CH}_{3}, 87\right), 161\left(\mathrm{M}^{+}-\mathrm{CH}_{3} \mathrm{O}, 93\right)$. Exact mass for $\mathrm{C}_{12} \mathrm{H}_{16} \mathrm{O}_{2} \mathrm{Ag}(\mathrm{M}+\mathrm{Ag})$ : calculated 299.0201, found 299.0202.

\section{1,3-Dimethyoxy-5-(2-methylpropenyl)benzene (Table 4, Entry 4)}<smiles>COc1cc(C=C(C)C)cc(OC)c1</smiles>

${ }^{1} \mathrm{H}$ NMR (400 MHz, $\left.\mathrm{CDCl}_{3}\right): \delta 6.37(\mathrm{~d}, \mathrm{~J}=2.29 \mathrm{~Hz}, 2 \mathrm{H}), 6.31(\mathrm{t}, \mathrm{J}=2.29 \mathrm{~Hz}, 1 \mathrm{H}), 6.20(\mathrm{~s}, 1$ H), 3.77 (s, $7 \mathrm{H}), 1.88(\mathrm{~s}, 3 \mathrm{H}), 1.86(\mathrm{~s}, 3 \mathrm{H})$. Spectral data are consistent with Table 4, Entry 3. 


\section{1-Cyclohexylidenemethyl-3,5-dimethoxybenzene (Table 4, Entry 5)}<smiles>COc1cc(C=C2CCCCC2)cc(OC)c1</smiles>

${ }^{1} \mathrm{H}$ NMR (400 MHz, $\left.\mathrm{CDCl}_{3}\right): \delta 6.35(\mathrm{~d}, \mathrm{~J}=2.29 \mathrm{~Hz}, 2 \mathrm{H}), 6.30(\mathrm{t}, \mathrm{J}=2.29 \mathrm{~Hz}, 1 \mathrm{H}), 6.15(\mathrm{~s}, 1$ $\mathrm{H}), 3.77(\mathrm{~s}, 6 \mathrm{H}), 2.40-2.34(\mathrm{~m}, 2 \mathrm{H}), 2.26-2.20(\mathrm{~m}, 2 \mathrm{H}), 1.66-1.52(\mathrm{~m}, 6 \mathrm{H}) .{ }^{13} \mathrm{C} \mathrm{NMR}(100$ $\left.\mathrm{MHz}, \mathrm{CDCl}_{3}\right): \delta 160.4,144.0,140.4,121.9,107.0,98.1,55.2,37.6,29.7,28.6,27.8,26.6$. Mass spectrum, m/z (relative intensity, \%): $232\left(\mathrm{M}^{+}, 100\right), 217\left(\mathrm{M}^{+}-\mathrm{CH}_{3}, 32\right), 201\left(\mathrm{M}^{+}-\mathrm{CH}_{3} \mathrm{O}, 75\right)$. Exact mass for $\mathrm{C}_{15} \mathrm{H}_{20} \mathrm{O}_{2} \mathrm{Ag}(\mathrm{M}+\mathrm{Ag})$ : calculated 339.0514, found 339.0508.

\section{Biphen-4-yldimethylamine (Table 4, Entry 6) ${ }^{10}$}<smiles>CN(C)c1ccc(-c2ccccc2)cc1</smiles>

${ }^{1} \mathrm{H}$ NMR (400 MHz, $\left.\mathrm{CDCl}_{3}\right): \delta 7.58(\mathrm{~d}, \mathrm{~J}=7.67 \mathrm{~Hz}, 2 \mathrm{H}), 7.53(\mathrm{~d}, \mathrm{~J}=8.29 \mathrm{~Hz}, 2 \mathrm{H}), 7.41(\mathrm{t}, \mathrm{J}=$ $7.67 \mathrm{~Hz}, 2 \mathrm{H}), 7.27$ (t, J = 7.28 Hz, $1 \mathrm{H}), 6.82$ (d, J = 8.29 Hz, $2 \mathrm{H}), 3.00$ (s, $6 \mathrm{H})$. Spectral data are consistent with literature data.

\section{(4-Cyclohexylidenemethylphenyl)dimethylamine (Table 4, Entry 7)}

\footnotetext{
${ }^{10}$ Najera, C., Gil-Molto, J., Karlstroem, S., Falvello, L. Org. Lett. 2003, 5, 1451.
} 


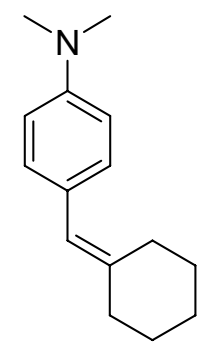

${ }^{1} \mathrm{H}$ NMR (400 MHz, $\left.\mathrm{CDCl}_{3}\right): \delta 7.12(\mathrm{~d}, \mathrm{~J}=8.30 \mathrm{~Hz}, 2 \mathrm{H}), 6.71(\mathrm{~d}, \mathrm{~J}=8.30 \mathrm{~Hz}, 2 \mathrm{H}), 6.16(\mathrm{~s}, 1$ H), 2.94 (s, 6 H), 2.44-2.38 (m, 2 H), 2.28-2.21 (m, $2 \mathrm{H}), 1.67-1.50(\mathrm{~m}, 4 \mathrm{H}) .{ }^{13} \mathrm{C}$ NMR $(100$ $\mathrm{MHz}, \mathrm{CDCl}_{3}$ ): $\delta 148.7,140.9,129.7,126.9,121.7,112.3,40.6,37.6,29.4,28.6,27.8,26.8$. Mass spectrum, m/z (relative intensity, \%): $215\left(100, \mathrm{M}^{+}\right), 200\left(10, \mathrm{M}^{+}-\mathrm{CH}_{3}\right)$. Exact mass for $\mathrm{C}_{15} \mathrm{H}_{21} \mathrm{NAg}(\mathrm{M}+\mathrm{Ag})$ : calculated 322.0725 , found 322.0718 .

\section{4-Trifluoromethylbiphenyl (Table 4, Entry 8) ${ }^{11}$}<smiles>FC(F)(F)c1ccc(-c2ccccc2)cc1</smiles>

${ }^{1} \mathrm{H}$ NMR (500 MHz, $\left.\mathrm{CDCl}_{3}\right): \delta 7.68(\mathrm{~s}, 4 \mathrm{H}), 7.58(\mathrm{~d}, \mathrm{~J}=7.55,2 \mathrm{H}), 7.46(\mathrm{t}, \mathrm{J}=7.37 \mathrm{~Hz}, 2 \mathrm{H})$, $7.39(\mathrm{~d}, \mathrm{~J}=7.37 \mathrm{~Hz}, 1 \mathrm{H})$ as a $4: 1$ mixture with $4,4^{\prime}$-bis(trifluoromethyl)biphenyl. Spectral data are consistent with literature data.

\section{1-(4-Cyclohexylidenemethylphenoxy)-2-phenoxyethane (Table 4, Entry 9)}<smiles>C(=C1CCCCC1)c1ccc(OCCOc2ccccc2)cc1</smiles>

${ }^{1} \mathrm{H}$ NMR (500 MHz, $\left.\mathrm{CDCl}_{3}\right)$ : $\delta$ 7.31-7.24 (m, $\left.2 \mathrm{H}\right)$, 7.14-7.06 (m, $\left.2 \mathrm{H}\right), 6.98-6.91(\mathrm{~m}, 3 \mathrm{H}), 6.91-$ $6.85(\mathrm{~m}, 2 \mathrm{H}), 6.15(\mathrm{~s}, 1 \mathrm{H}), 4.31(\mathrm{~s}, 4 \mathrm{H}), 2.34(\mathrm{t}, \mathrm{J}=5.89 \mathrm{~Hz}, 2 \mathrm{H}), 2.22(\mathrm{t}, \mathrm{J}=5.89 \mathrm{~Hz}, 2 \mathrm{H})$,

\footnotetext{
${ }^{11}$ Ueura, K.; Satoh, T.; Miura, M. Org. Lett. 2005, 11, 2229.
} 
1.65-1.49 (m, $6 \mathrm{H}) .{ }^{13} \mathrm{C}$ NMR (125 MHz, $\left.\mathrm{CDCl}_{3}\right): \delta 158.6,156.7,142.4,131.4,130.0,129.5$, 121.3, 121.1, 114.7, 114.3, 66.5, 66.4, 37.6, 29.4, 28.6, 27.8, 26.7. Mass spectrum, m/z (relative intensity, \%): $308\left(100, \mathrm{M}^{+}\right), 187\left(19, \mathrm{M}^{+}-\mathrm{C}_{6} \mathrm{H}_{5} \mathrm{OC}_{2} \mathrm{H}_{4}\right)$. Exact mass for $\mathrm{C}_{21} \mathrm{H}_{24} \mathrm{O}_{2} \mathrm{Ag}(\mathrm{M}+\mathrm{Ag})$ : calculated 415.0827, found 415.0826 amu.

1,2-diphenoxyethane (3) 
<smiles>c1ccc(OCCOc2ccccc2)cc1</smiles>

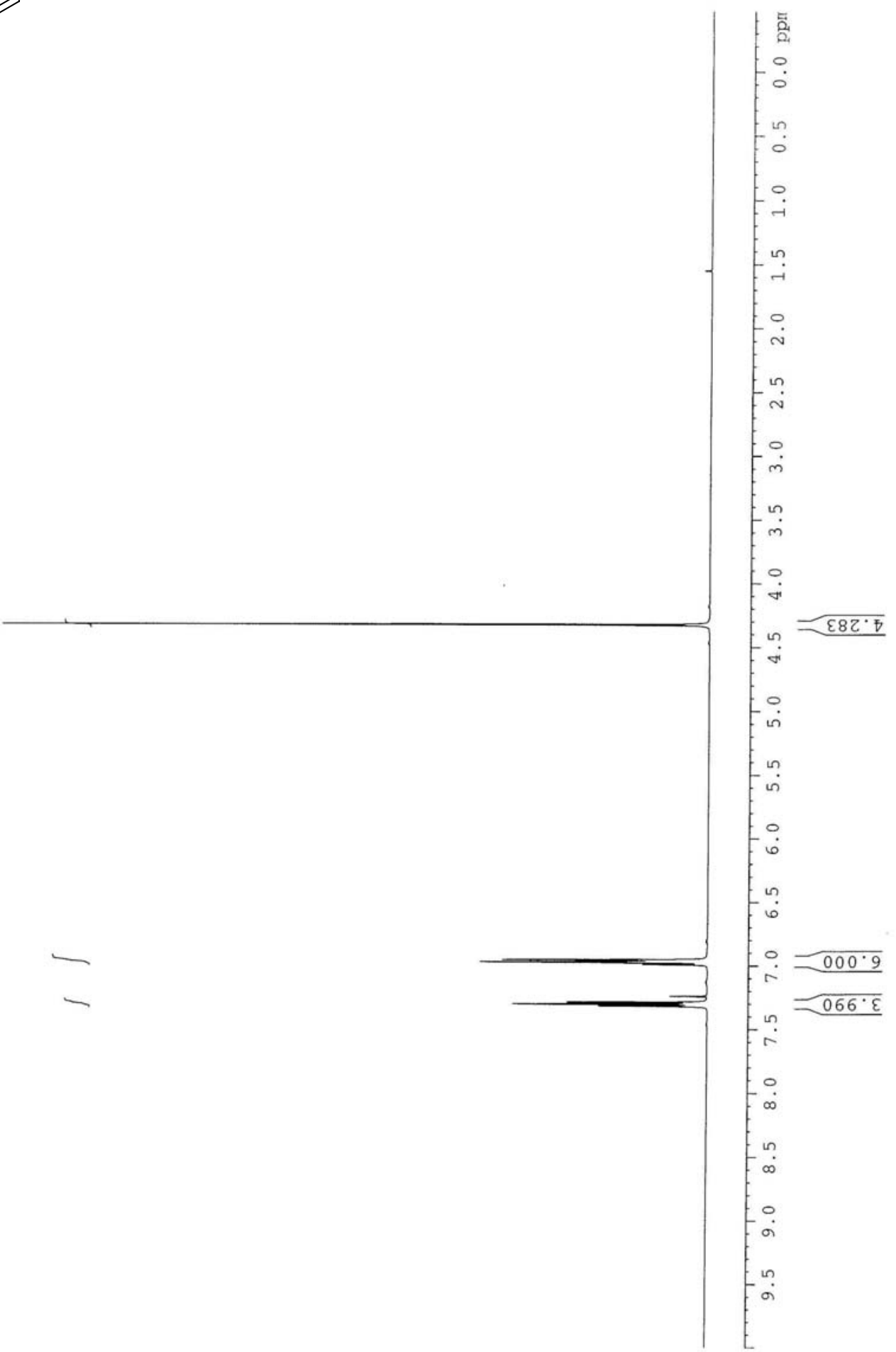

1-(Biphen-4-yloxy)-2-phenoxyethane (4) 

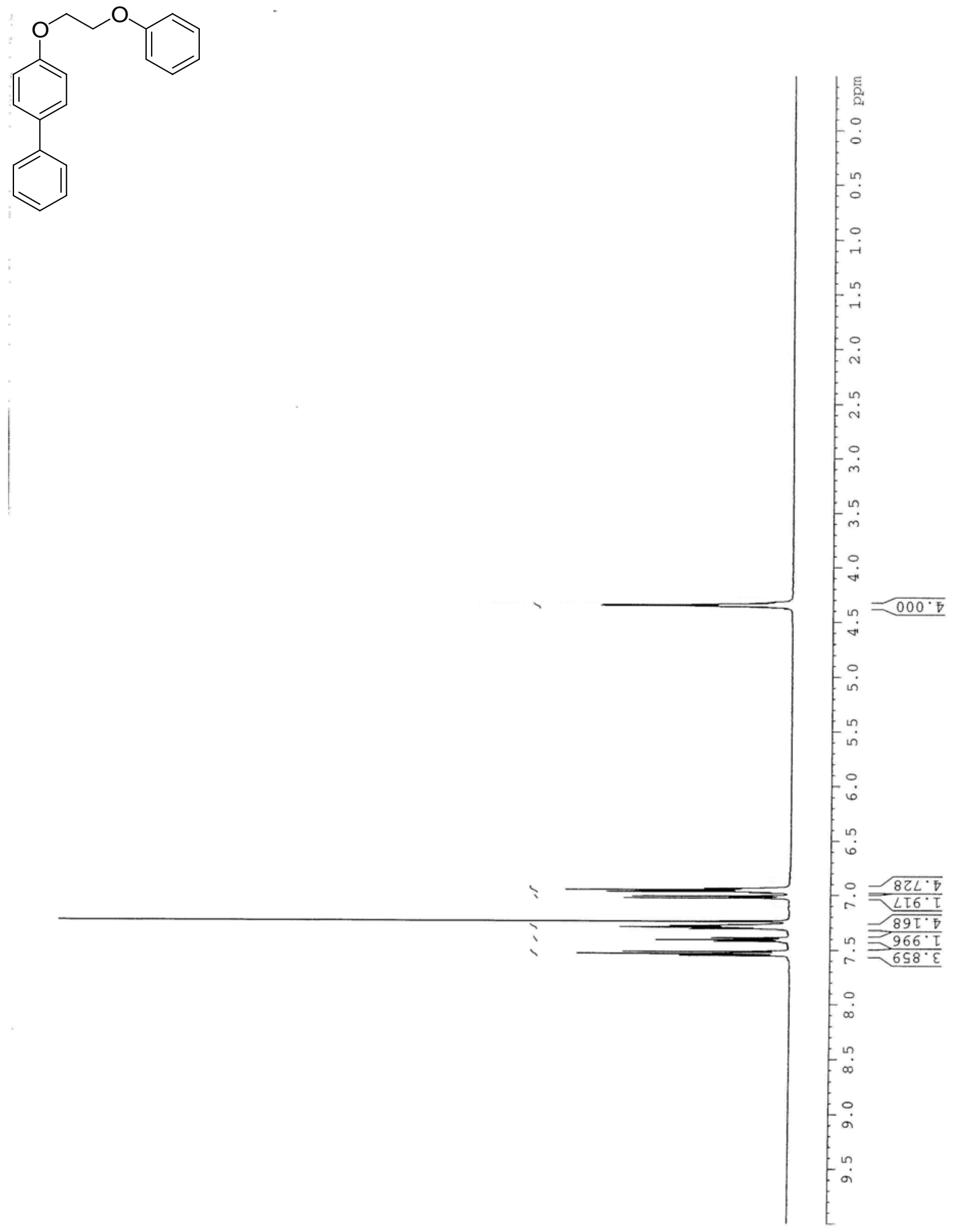

1-(Biphen-4-yloxy)-2-phenoxyethane (4) cont... 


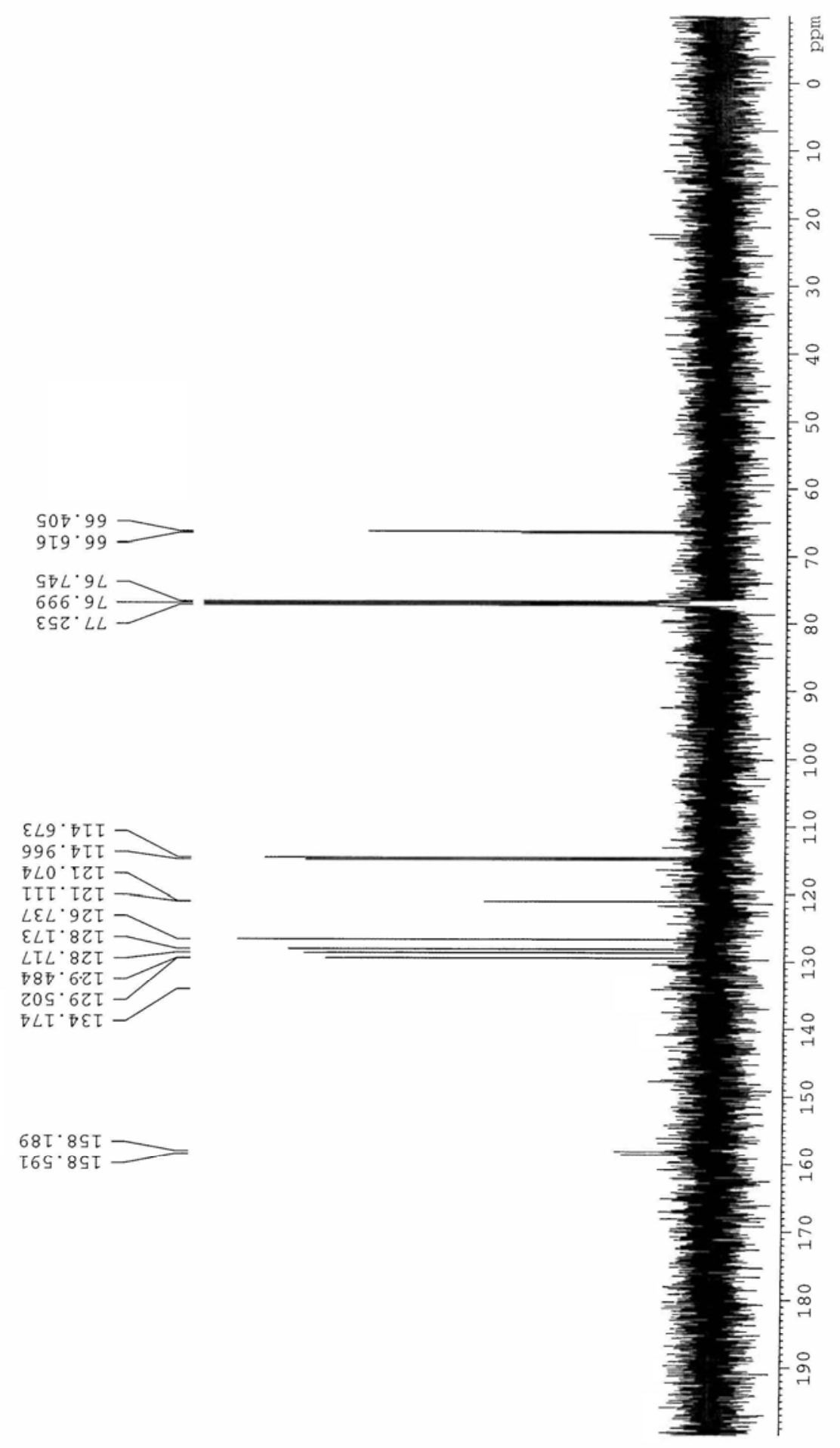

1-(Biphen-4-yloxy)-2-phenoxyethane (4) cont... 

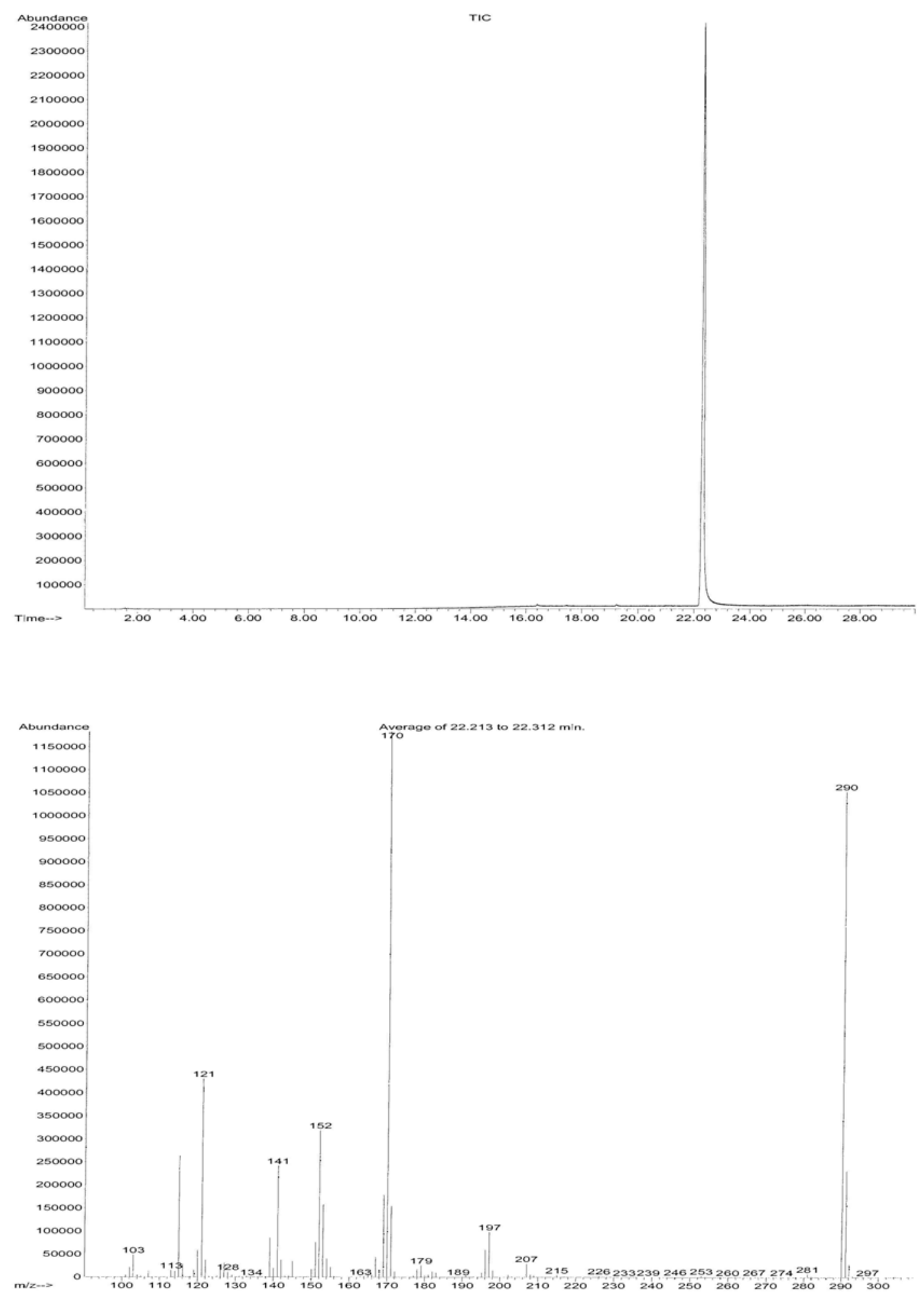

\section{4-Methoxybiphenyl (7)}


<smiles>COc1ccc(-c2ccccc2)cc1</smiles>

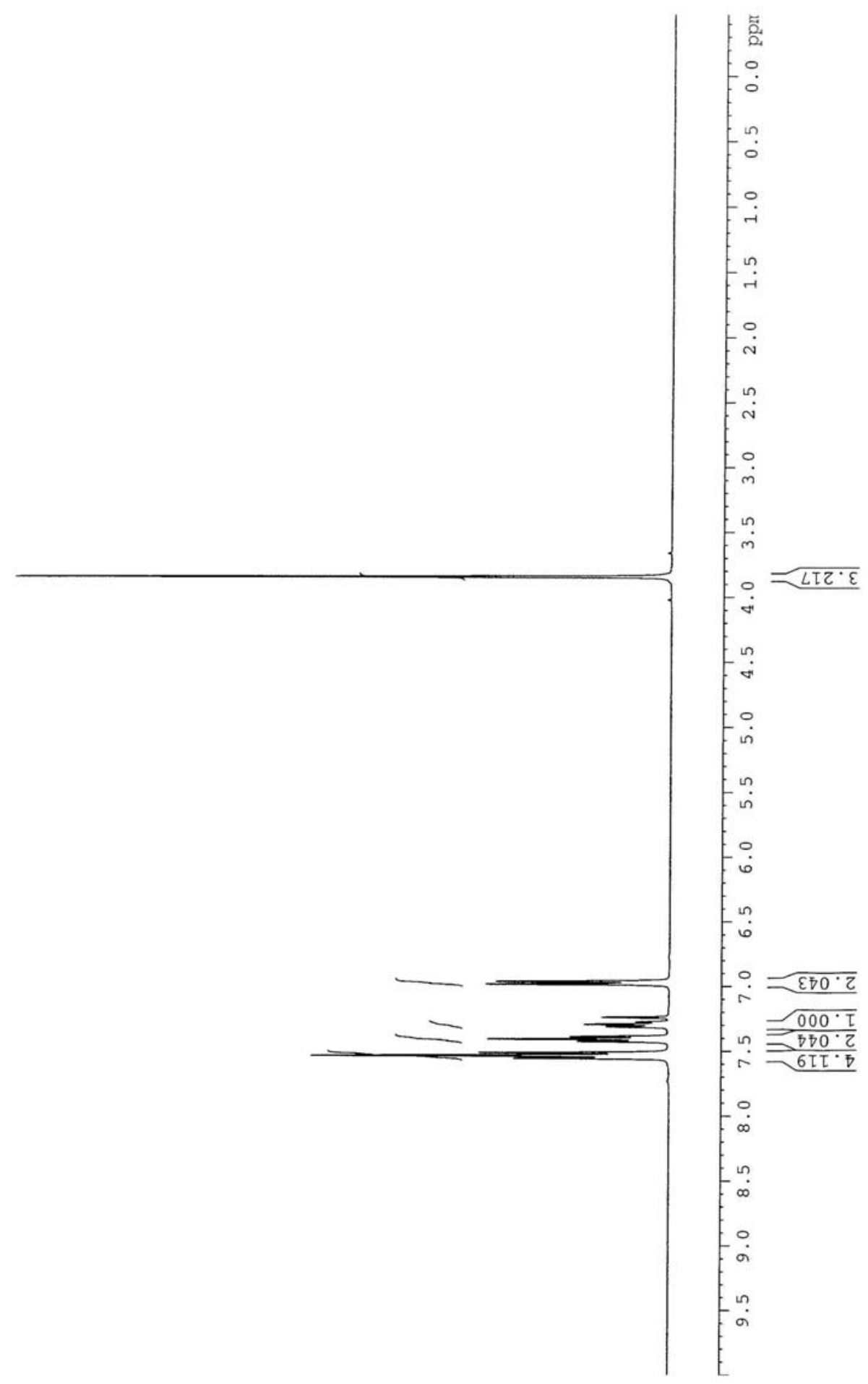

4,4'-Dimethoxybiphenyl (8) 
<smiles>COc1ccc(-c2ccc(OC)cc2)cc1</smiles>

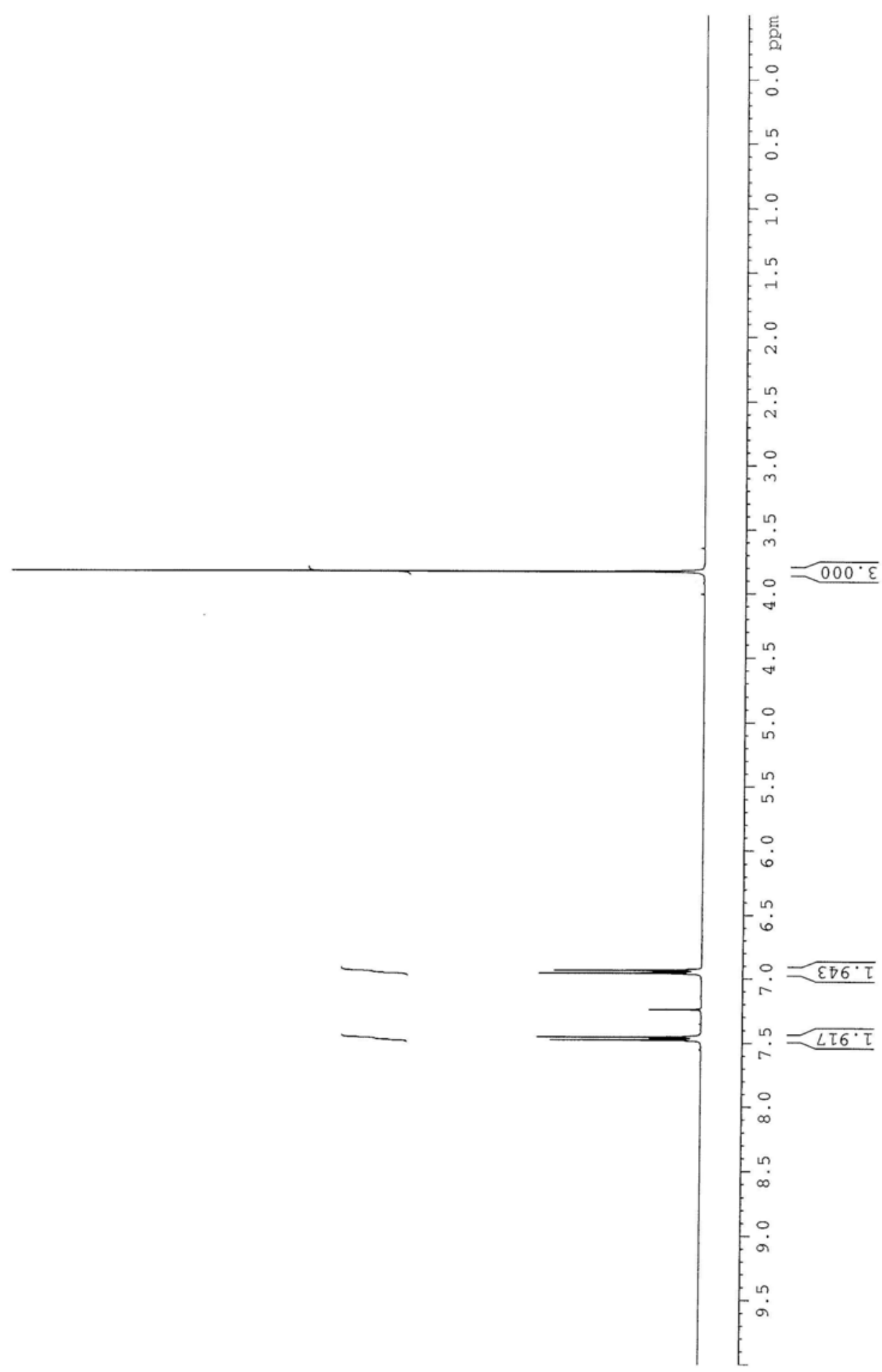

4-Methoxybiphenyl (Table 3, Entry 1) 
<smiles>COc1ccc(-c2ccccc2)cc1</smiles>

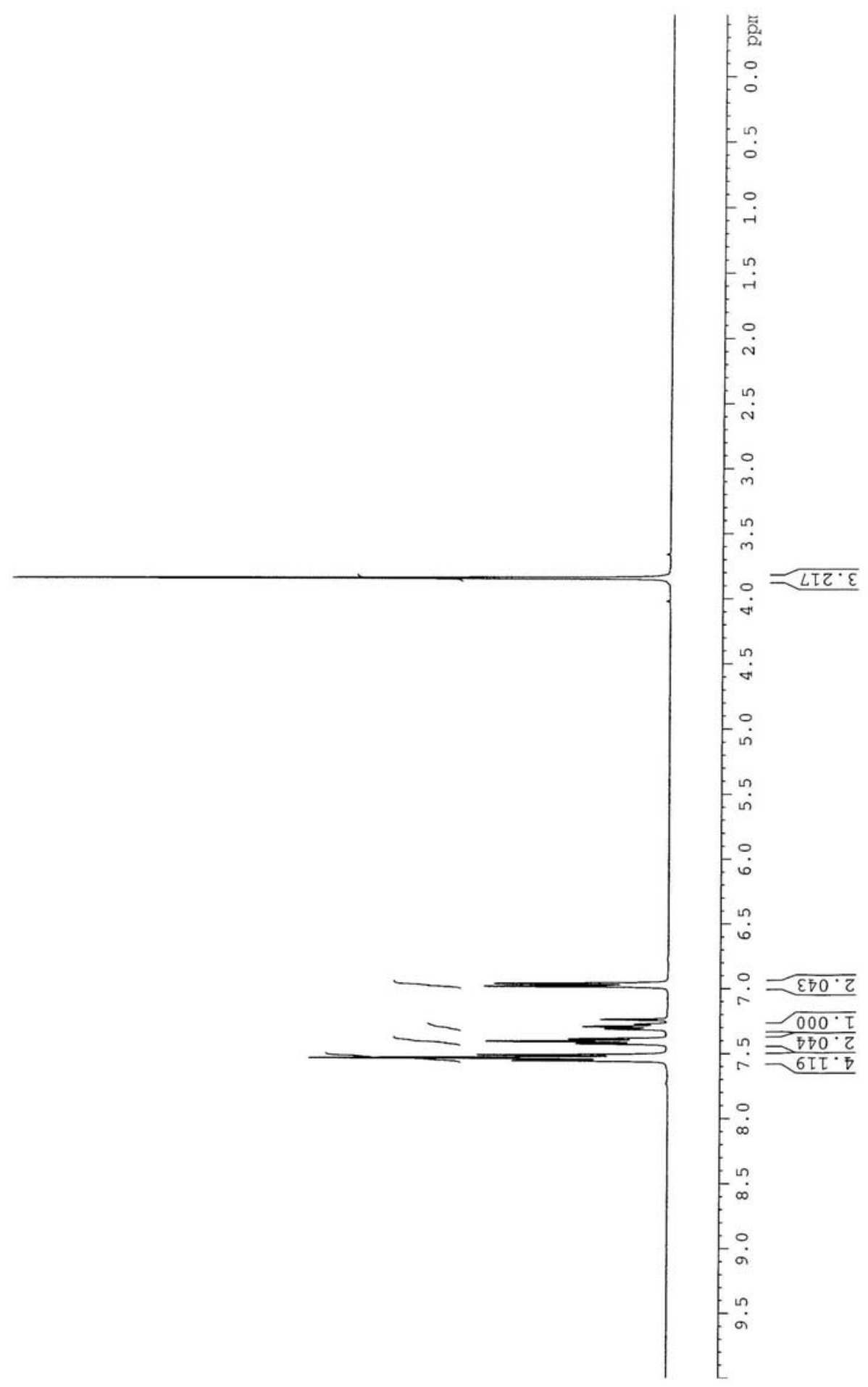

4-Methoxybiphenyl (Table 3, Entry 2)

OMe

1 


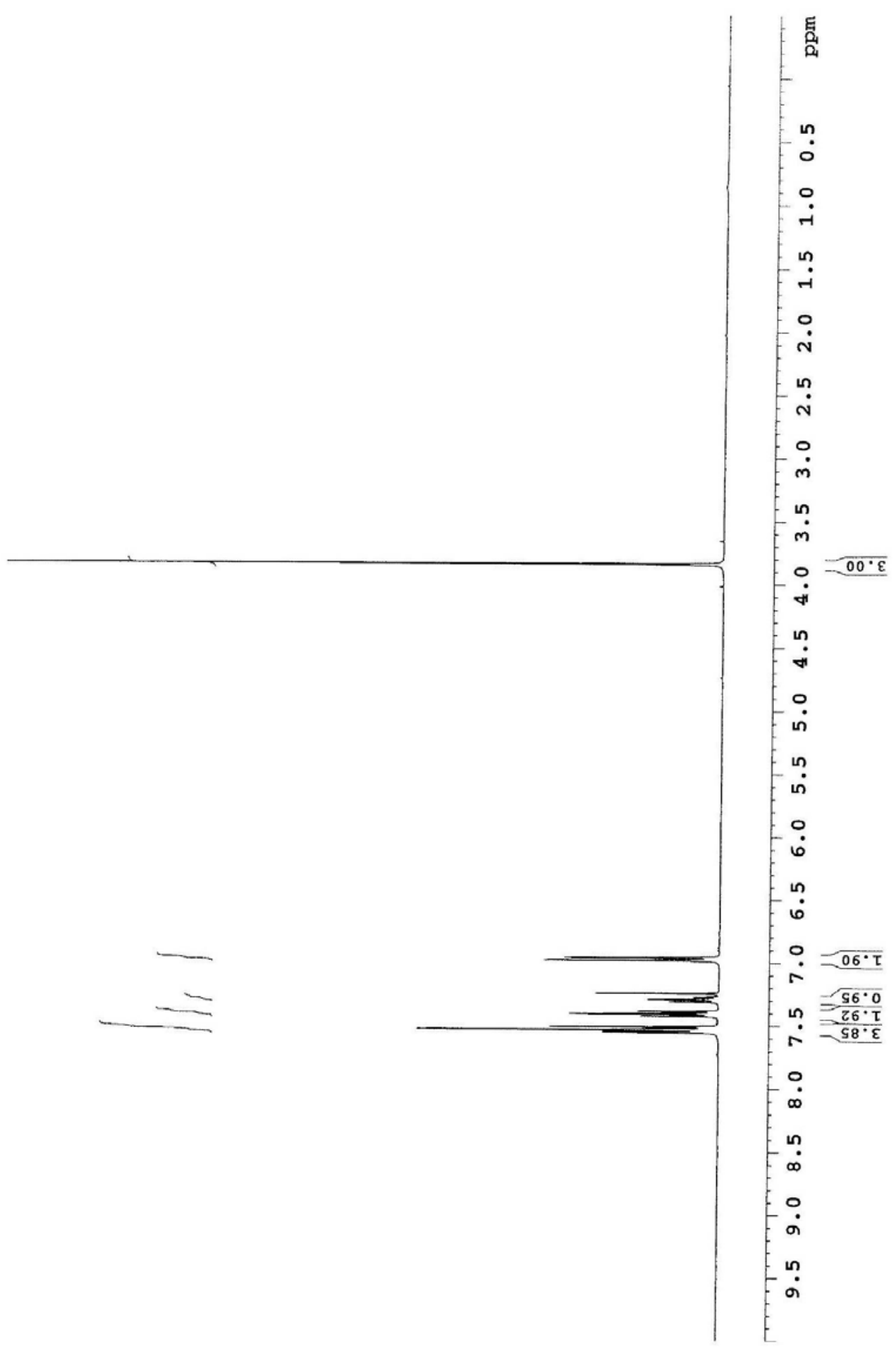

4-Methoxybiphenyl (Table 3, Entry 3)

OMe

1 


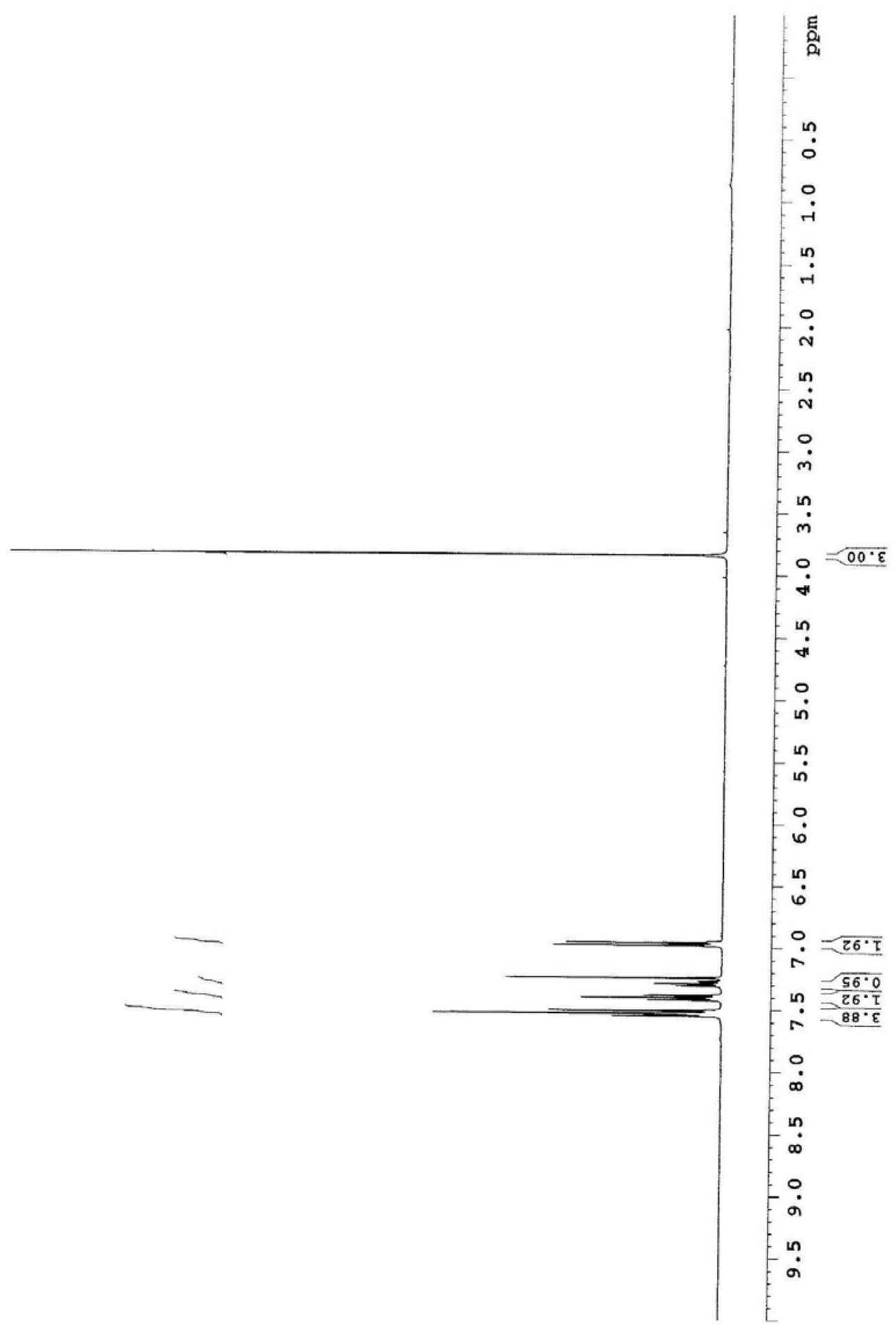

4-Methoxybiphenyl (Table 3, Entry 4)

OMe

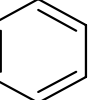




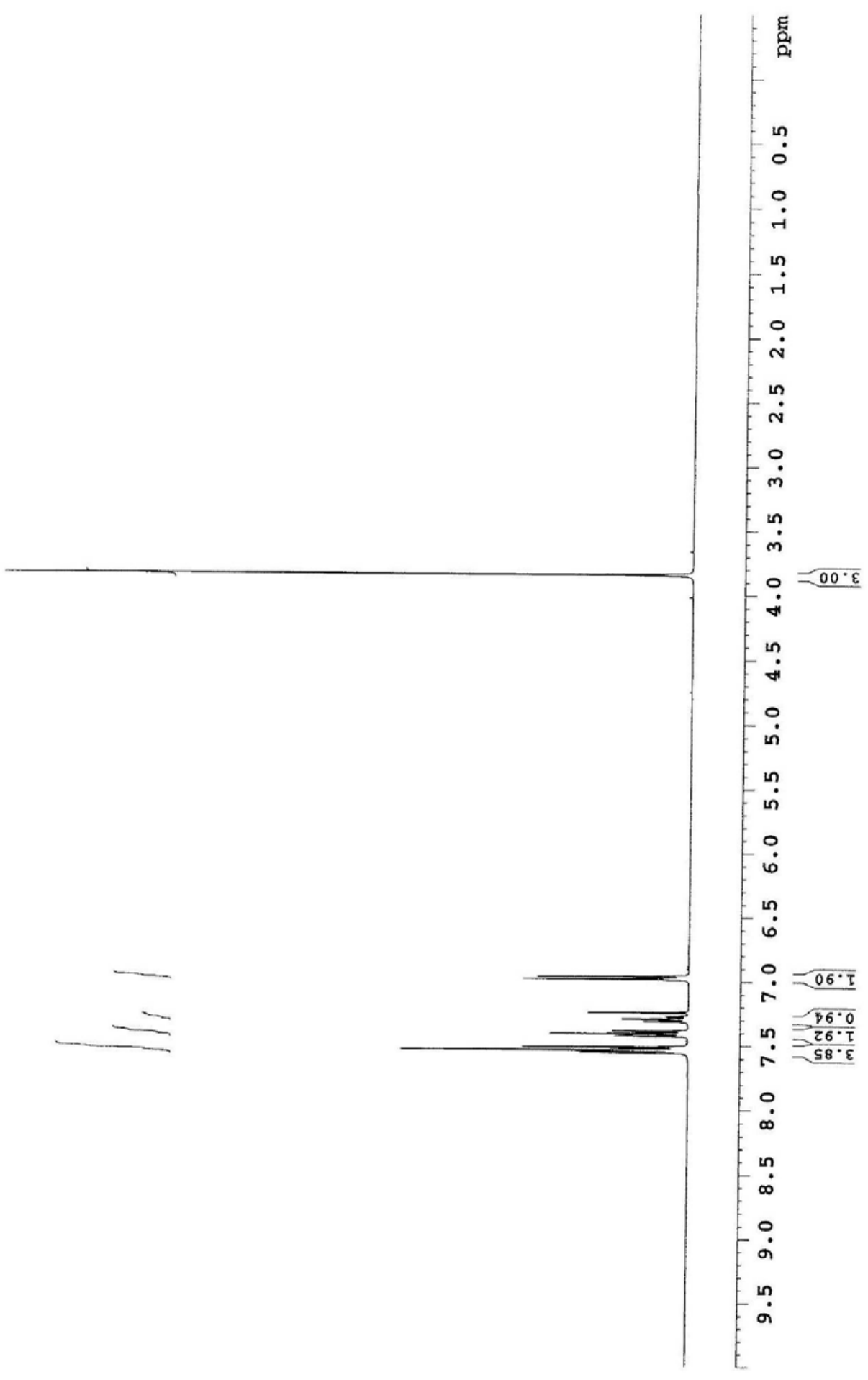

1-Methoxy-4-(2-propenyl)benzene (Table 3, Entry 5) 

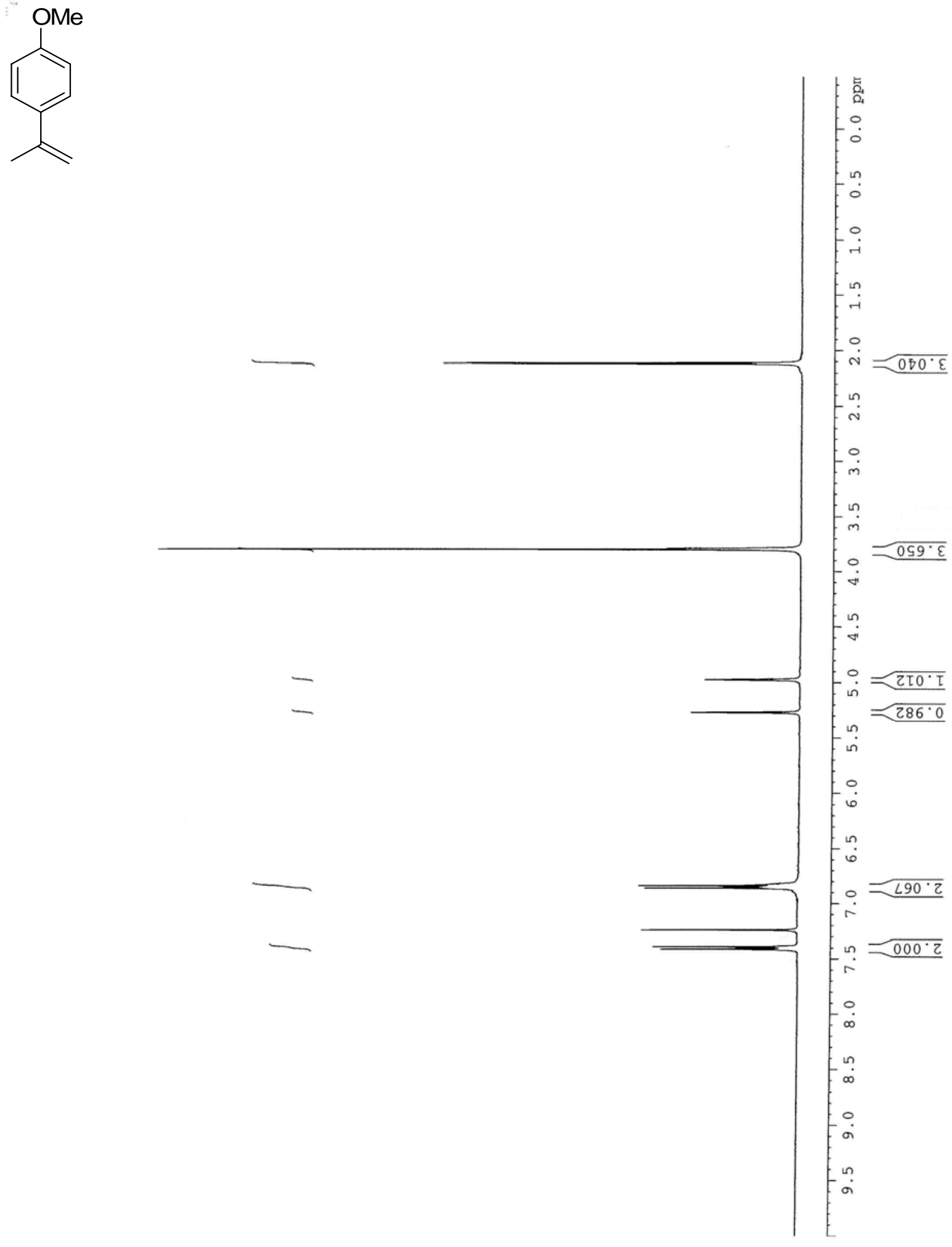
1-Cyclohexylidenemethyl-4-methoxybenzene (Table 3, Entry 6)<smiles>COc1ccc(C=C2CCCCC2)cc1</smiles>

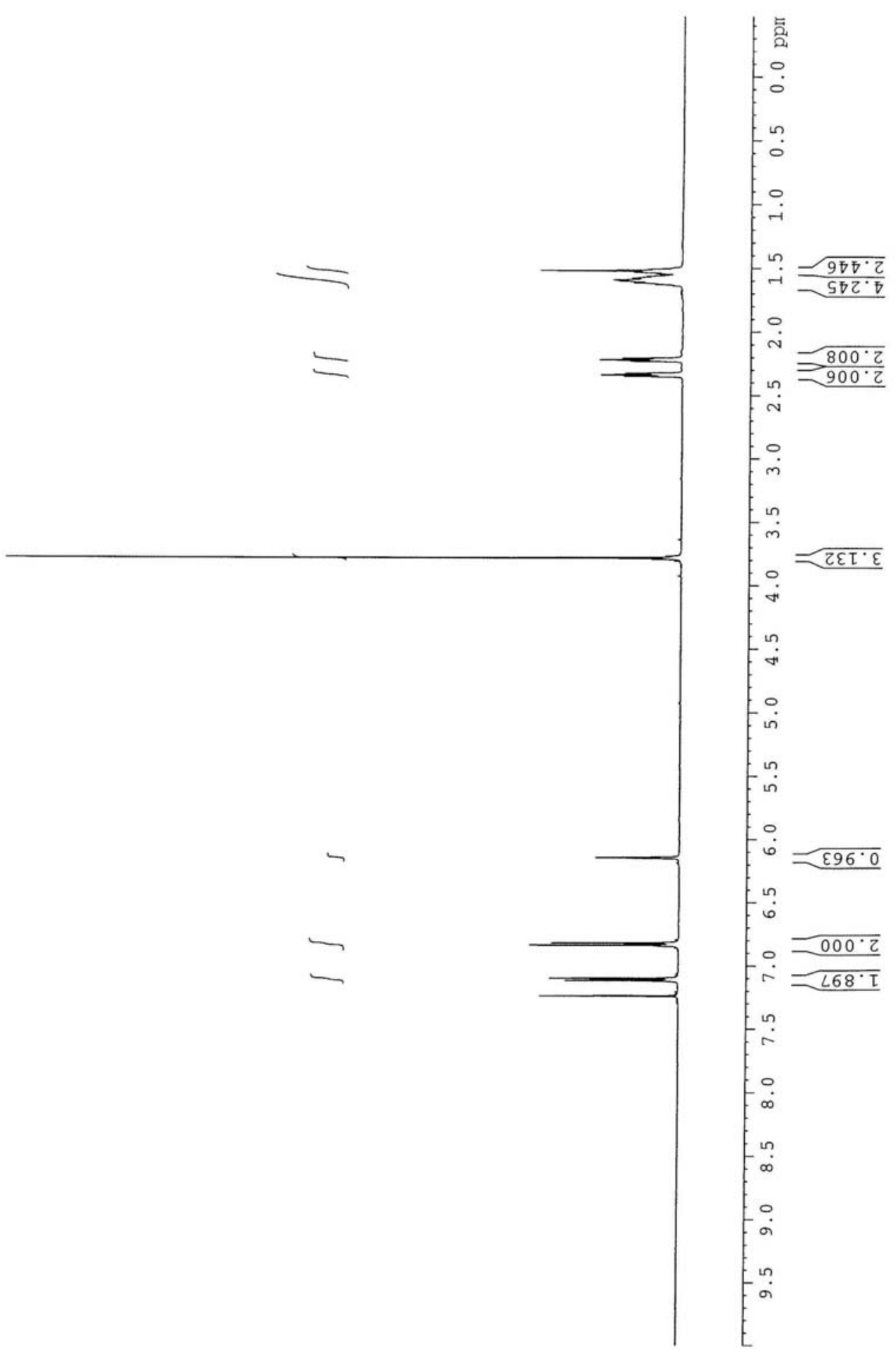


1-Methoxy-4-(2-methylpropenyl)benzene (Table 3, Entry 7)<smiles>COc1ccc(C=C(C)C)cc1</smiles>

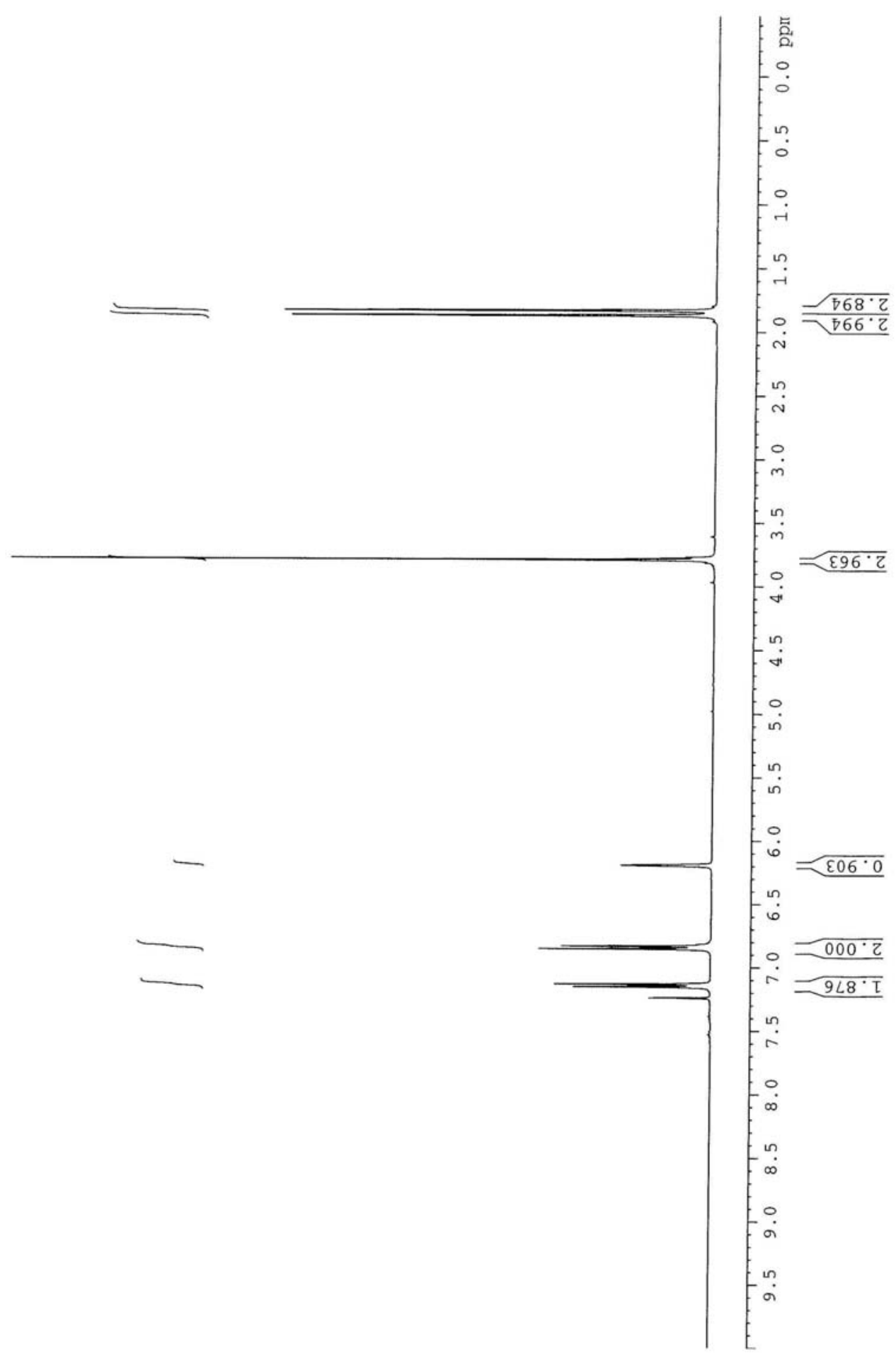


1-Methoxy-4-(2-methylpropenyl)benzene (Table 3, Entry 8)

COMe

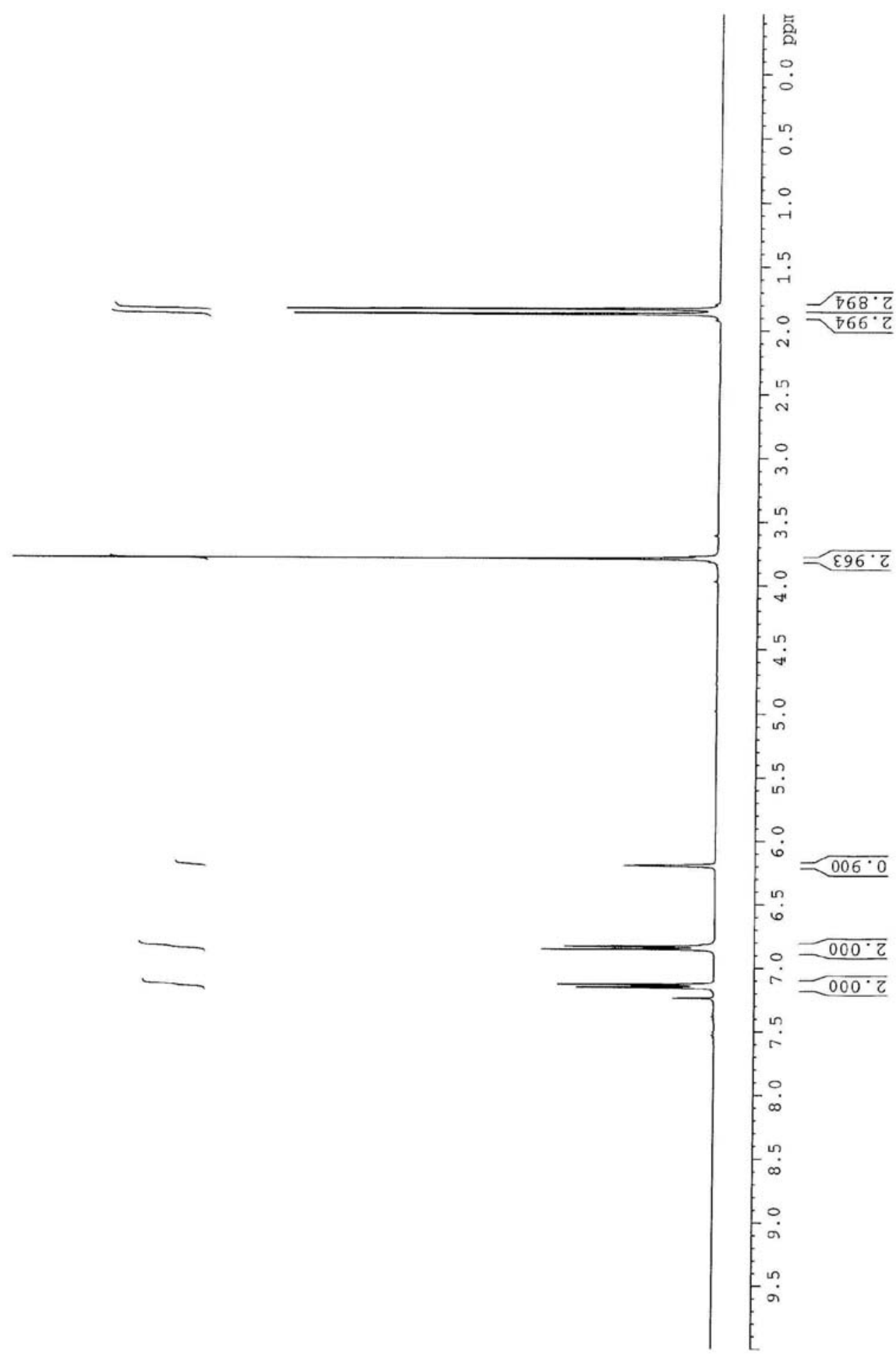


2-(4-Methoxyphenyl)pyridine (Table 3, Entry 9)<smiles>COc1ccc(-c2ccccn2)cc1</smiles>

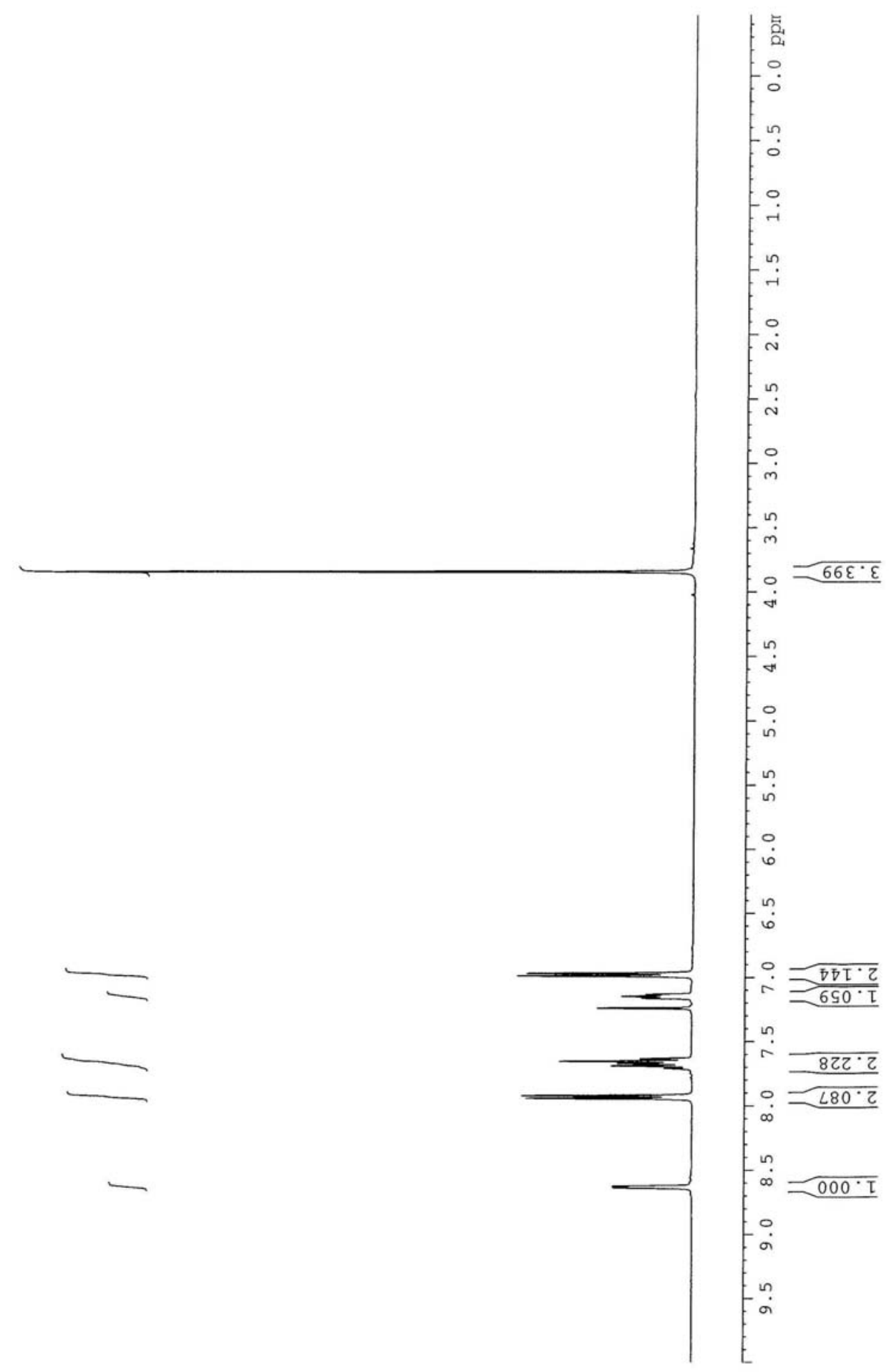


4-methoxy-4'-trifluoromethylbiphenyl (Table 3, Entry 10)<smiles>COc1ccc(-c2ccc(C(F)(F)F)cc2)cc1</smiles>

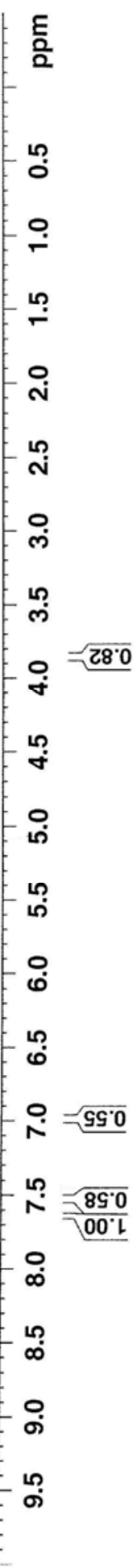


3,5-Dimethoxybiphenyl (Table 4, Entry 1)
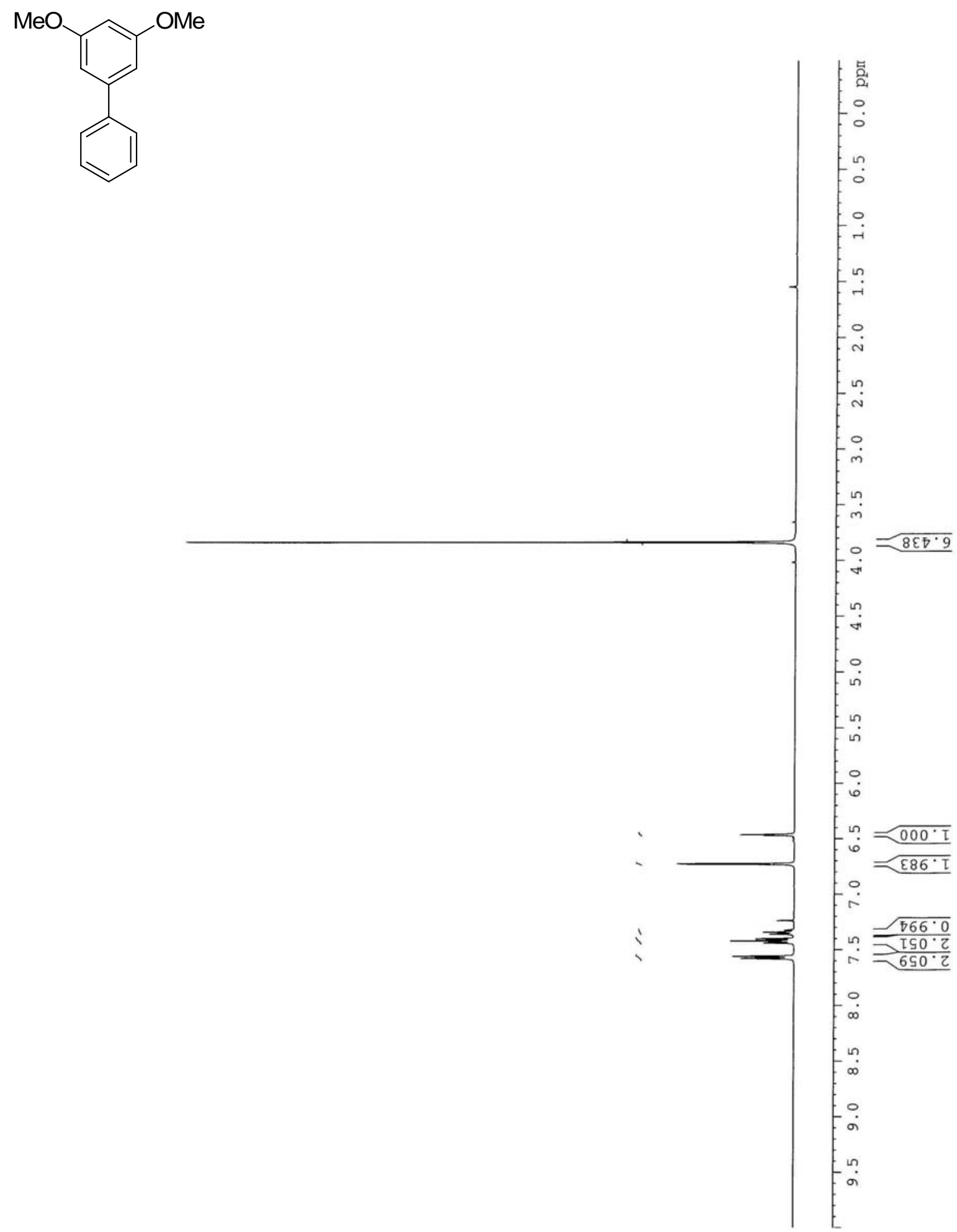
1,3-dimethoxy-5-(2-propenyl)benzene (Table 4, Entry 2)

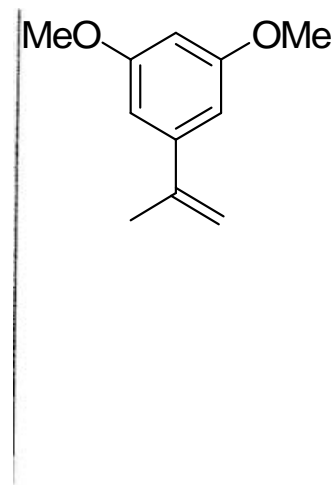

$-$

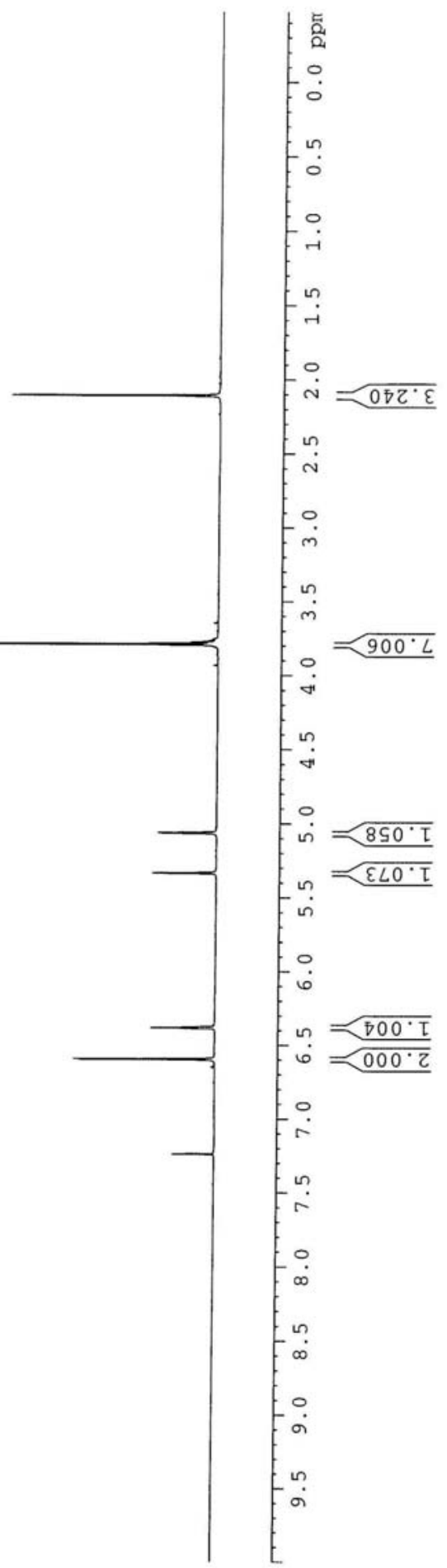


1,3-dimethoxy-5-(2-propenyl)benzene (Table 4, Entry 2) cont.

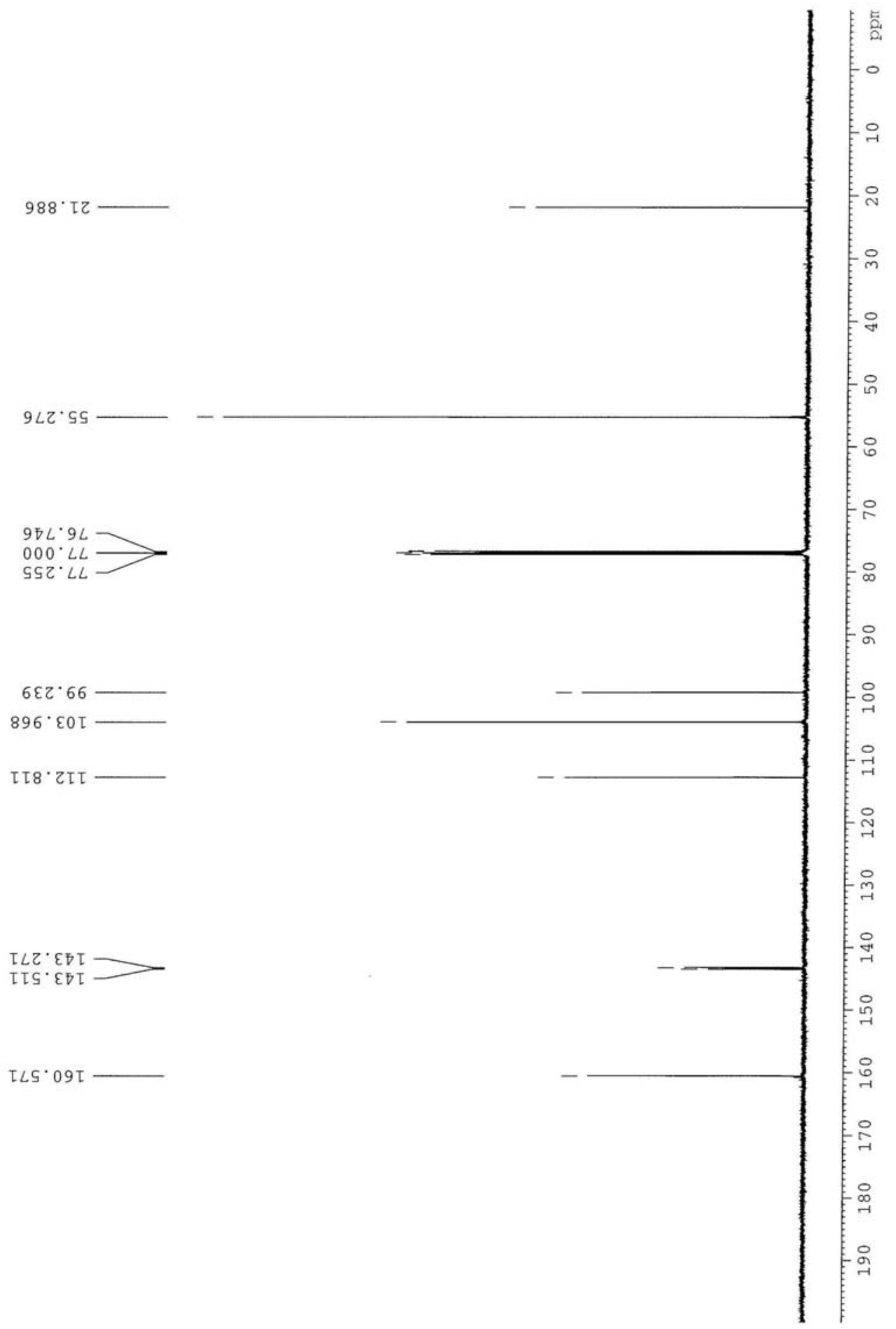




\section{1,3-dimethoxy-5-(2-propenyl)benzene (Table 4, Entry 2) cont.}

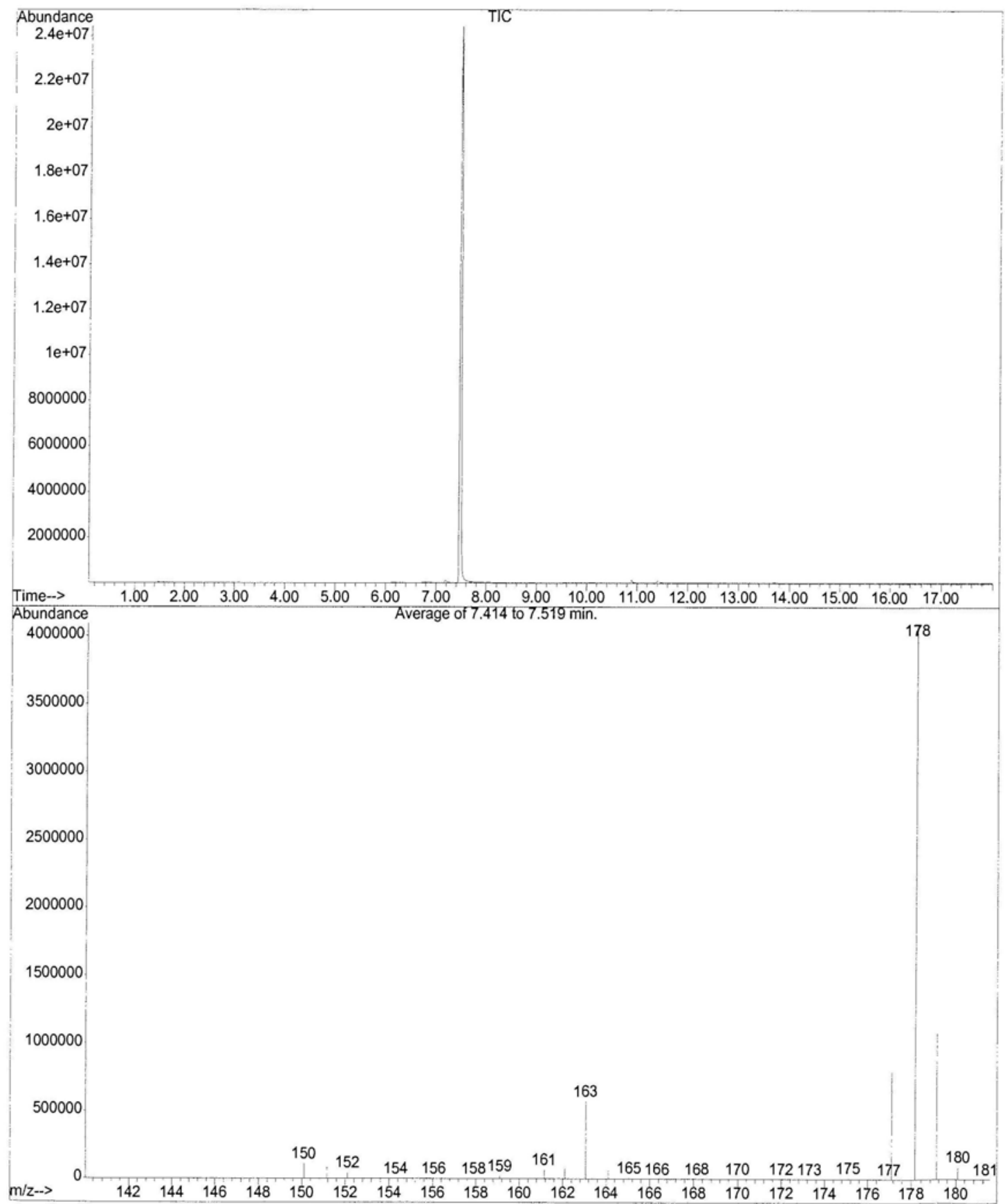


1,3-Dimethyoxy-5-(2-methylpropenyl)benzene (Table 4, Entry 3)
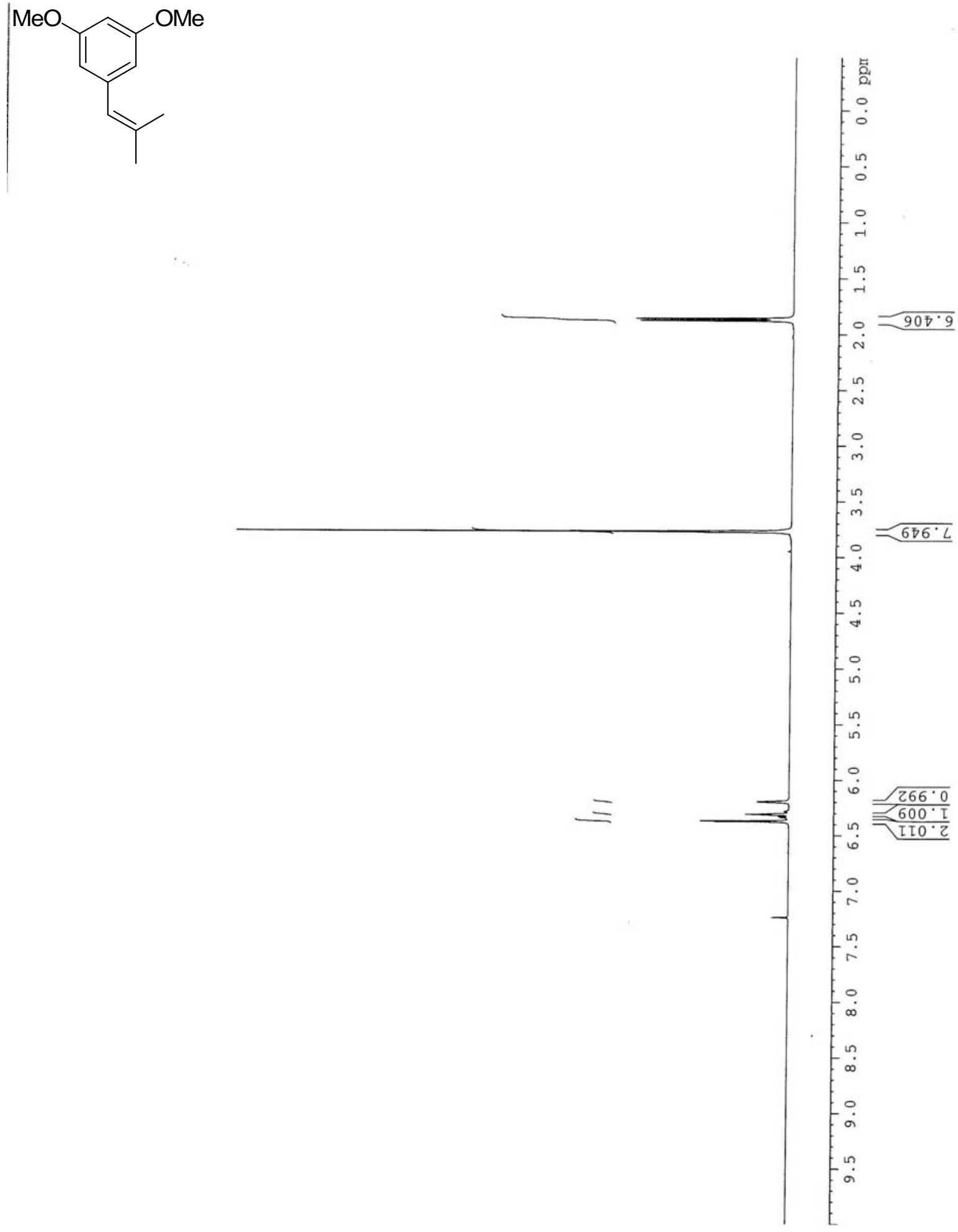
1,3-Dimethyoxy-5-(2-methylpropenyl)benzene (Table 4, Entry 3) cont.

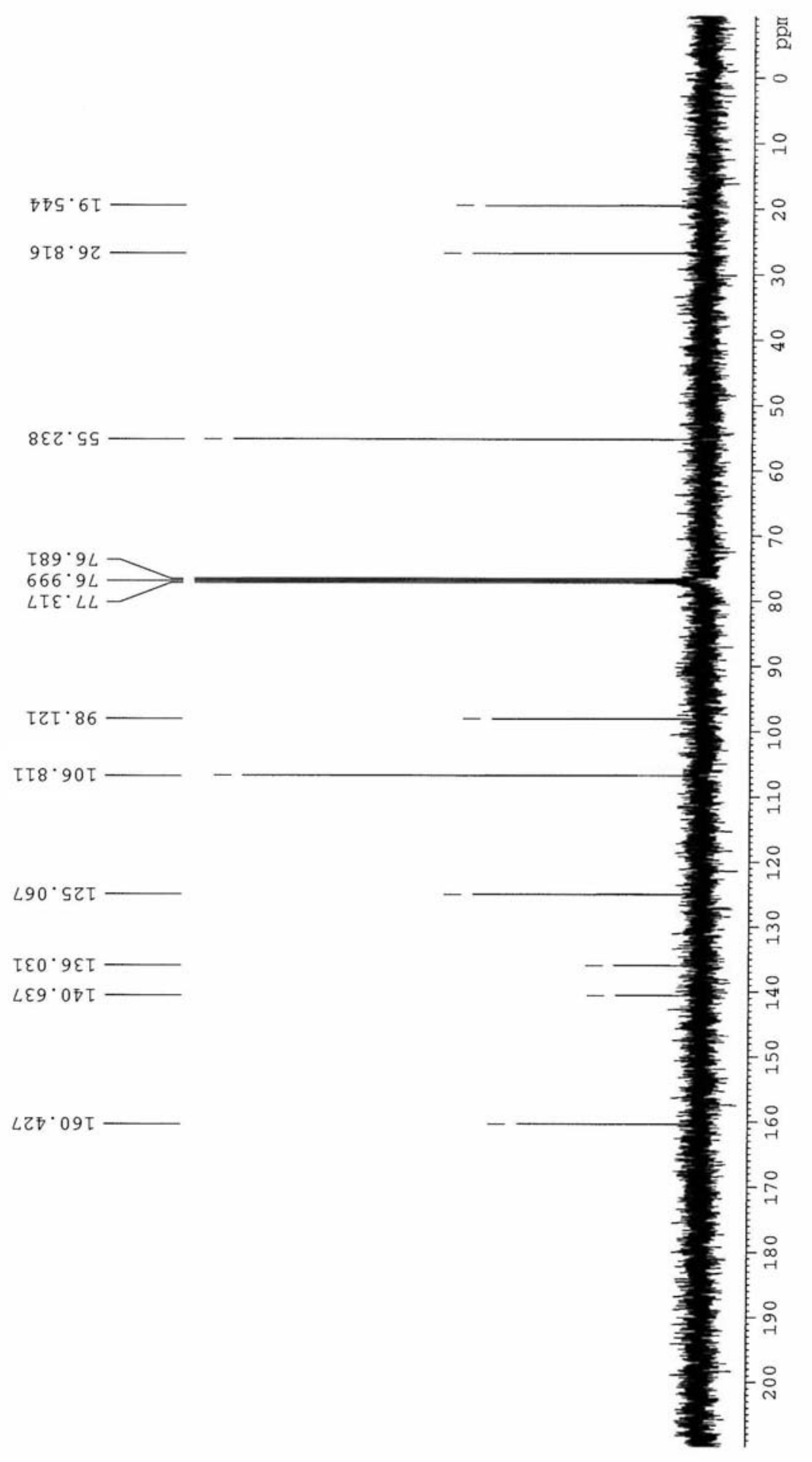




\section{1,3-Dimethyoxy-5-(2-methylpropenyl)benzene (Table 4, Entry 3) cont.}

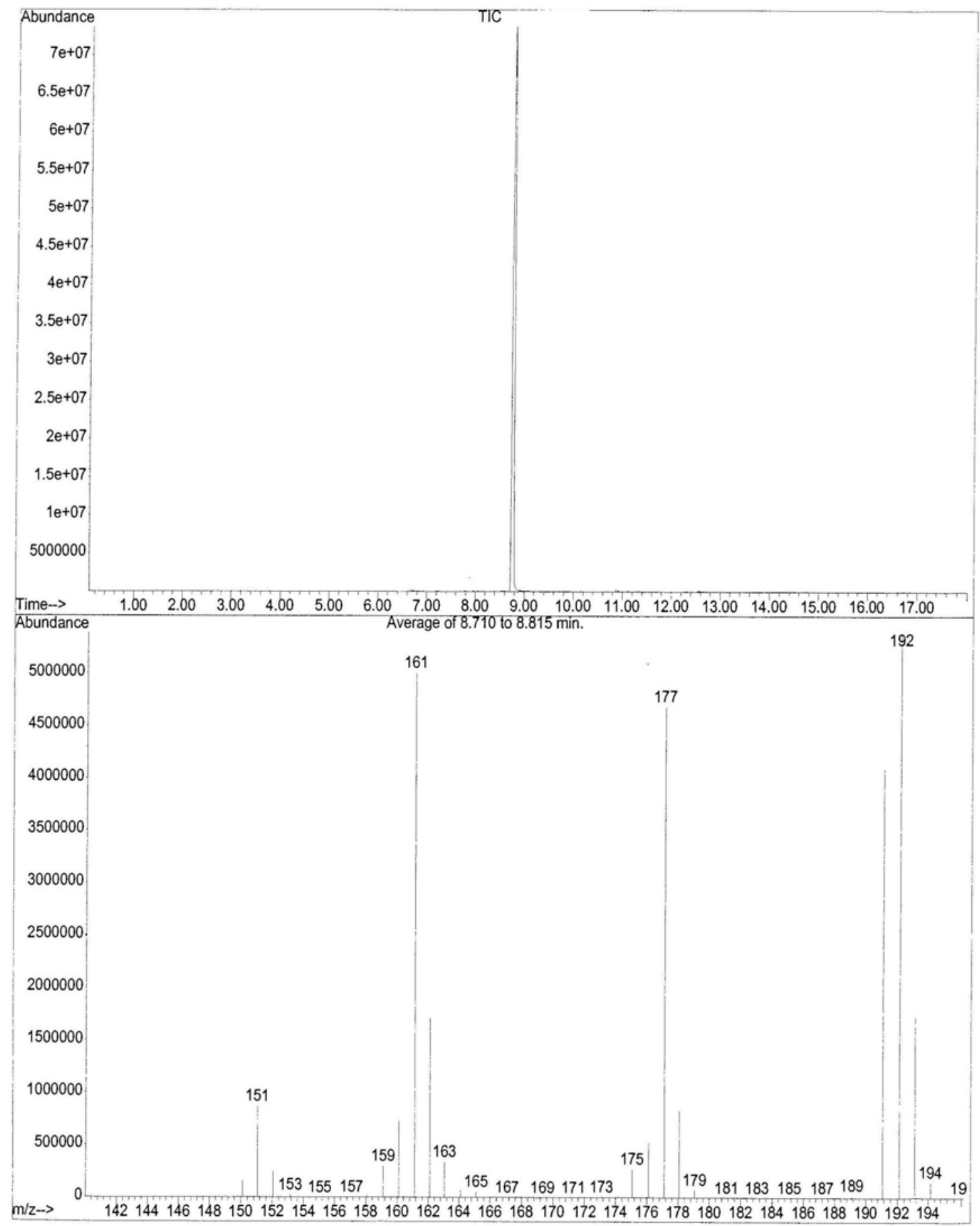


1,3-Dimethyoxy-5-(2-methylpropenyl)benzene (Table 4, Entry 4)
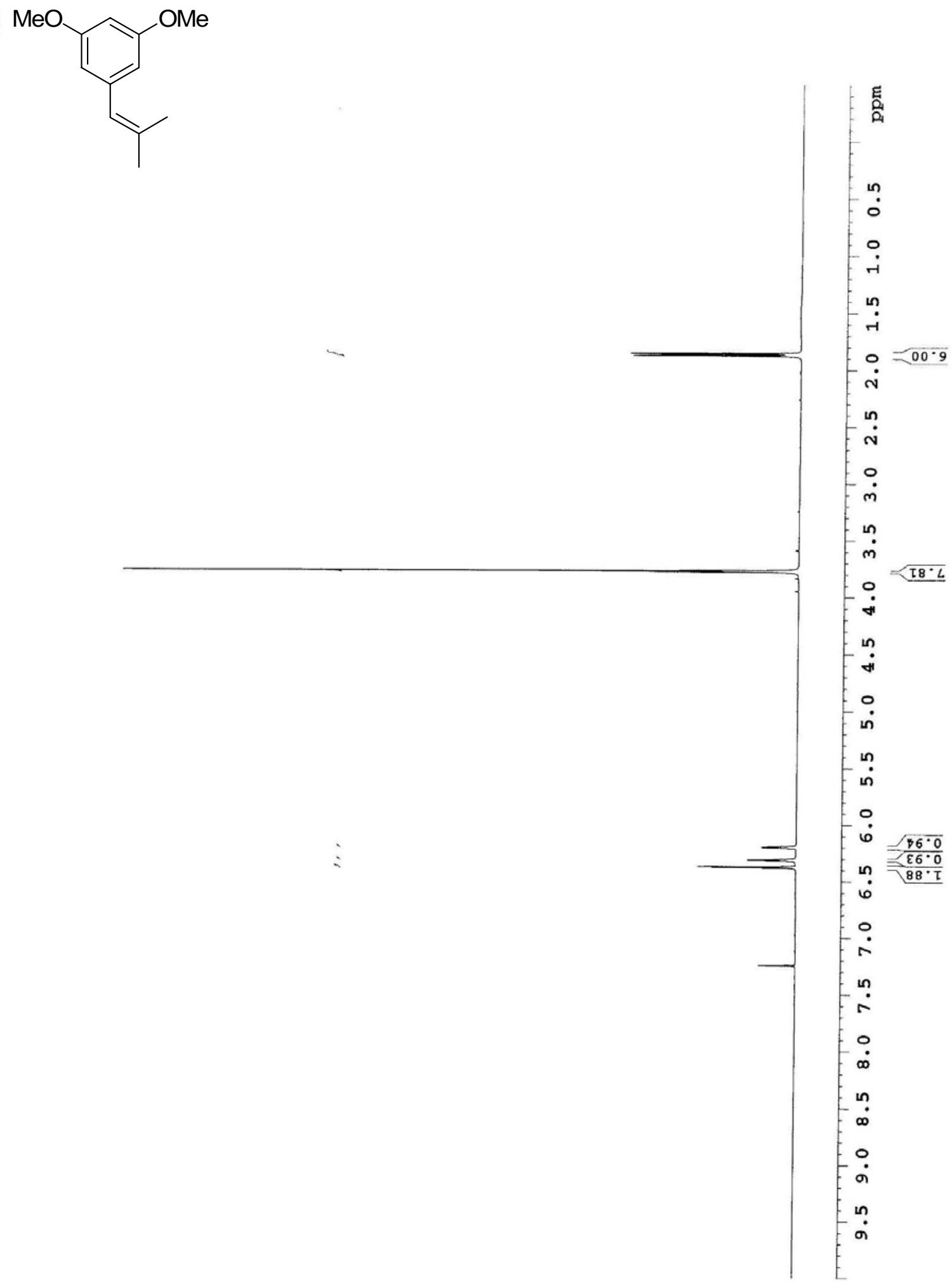
1-Cyclohexylidenemethyl-3,5-dimethoxybenzene (Table 4, Entry 5)<smiles>COc1cc(C=C2CCCCC2)cc(OC)c1</smiles>

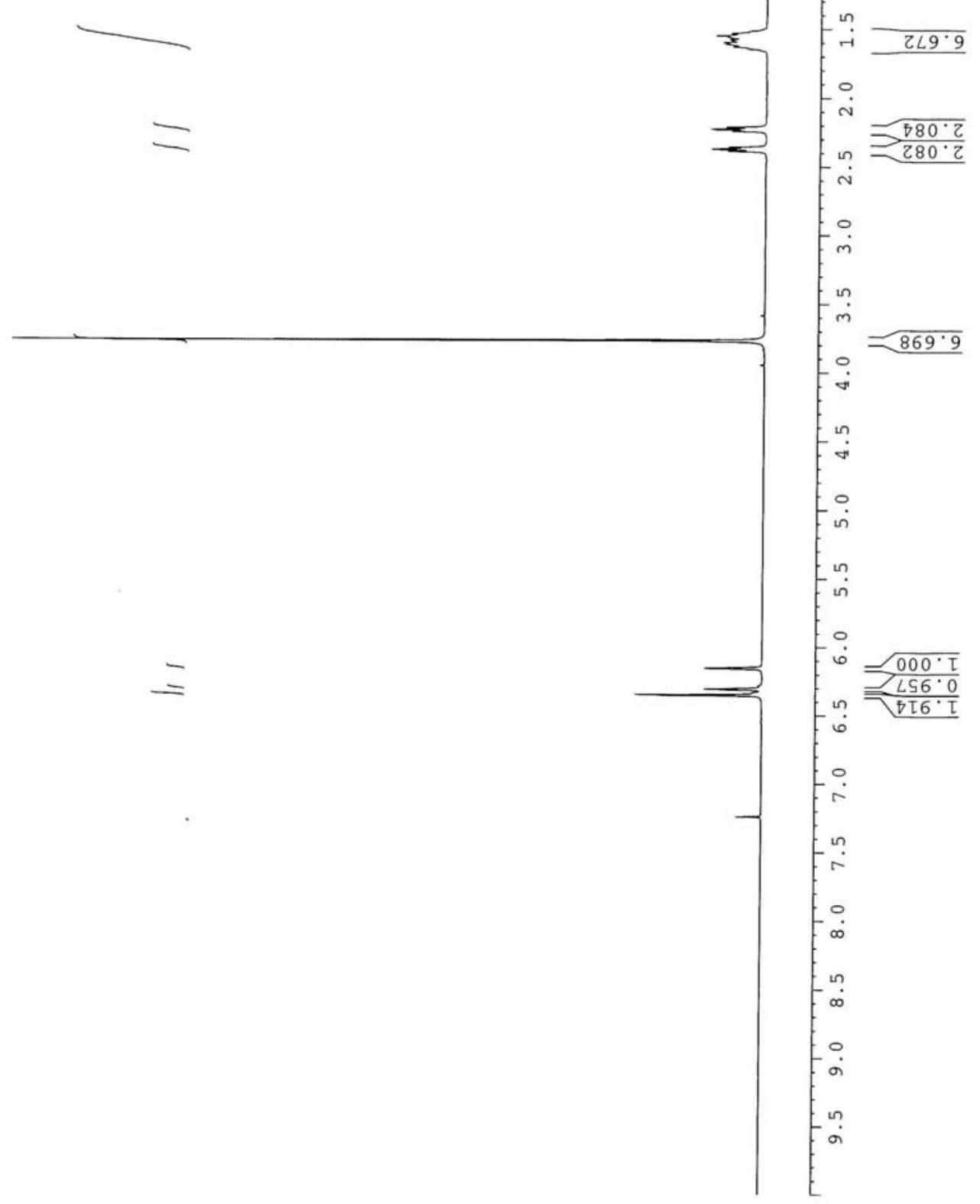


1-Cyclohexylidenemethyl-3,5-dimethoxybenzene (Table 4, Entry 5) cont.

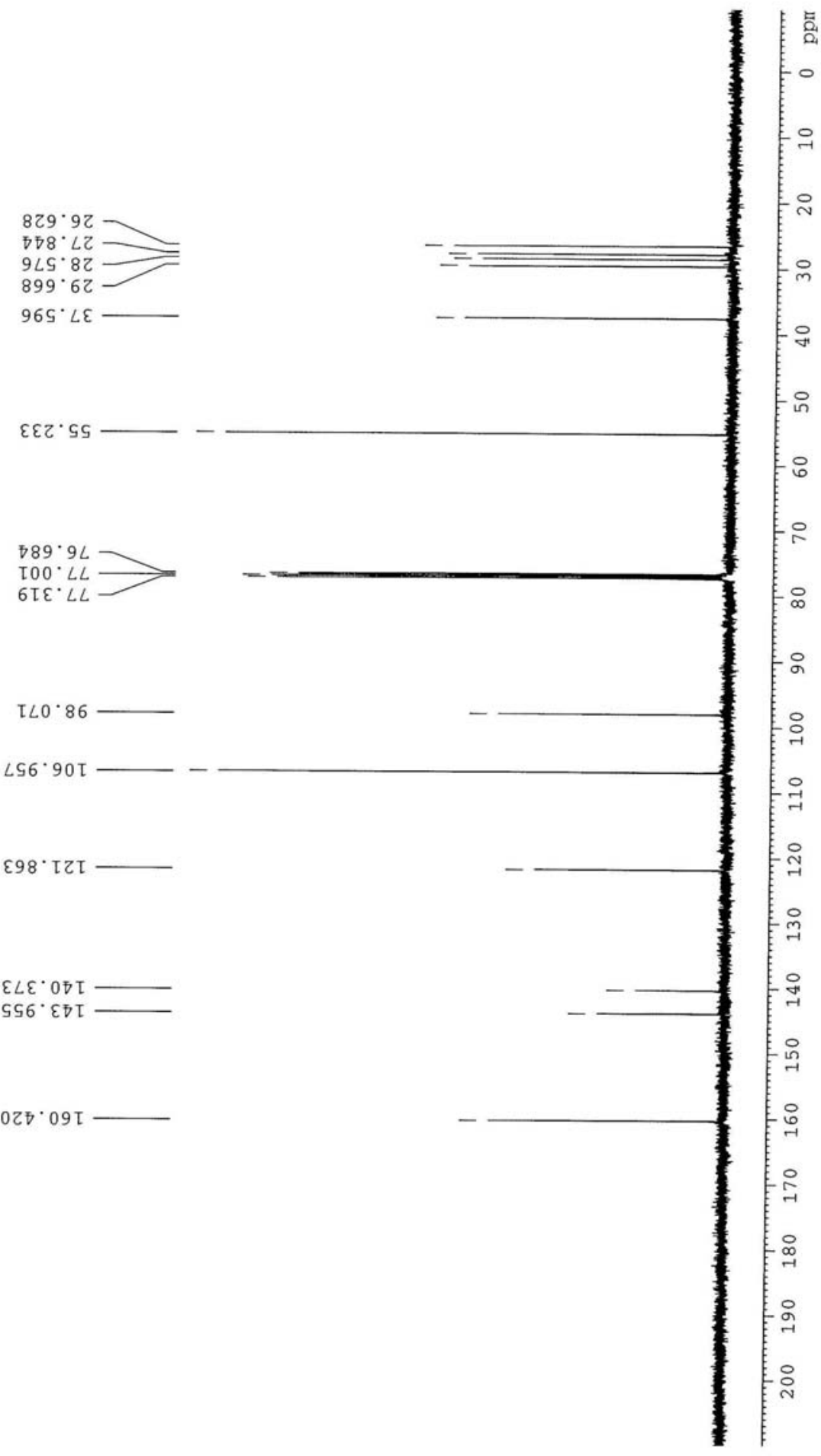




\section{1-Cyclohexylidenemethyl-3,5-dimethoxybenzene (Table 4, Entry 5) cont.}

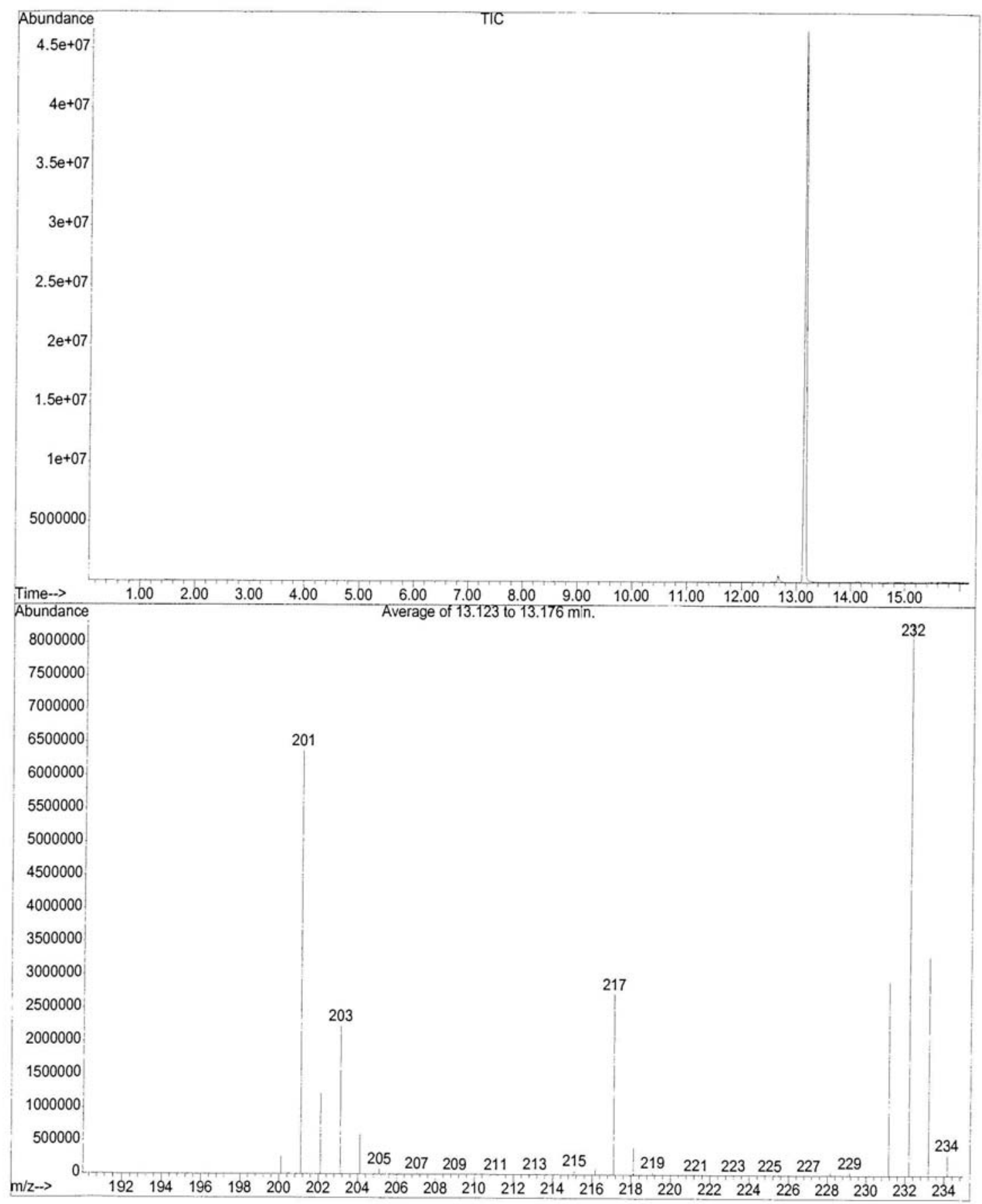


Biphen-4-yldimethylamine (Table 4, Entry 6)

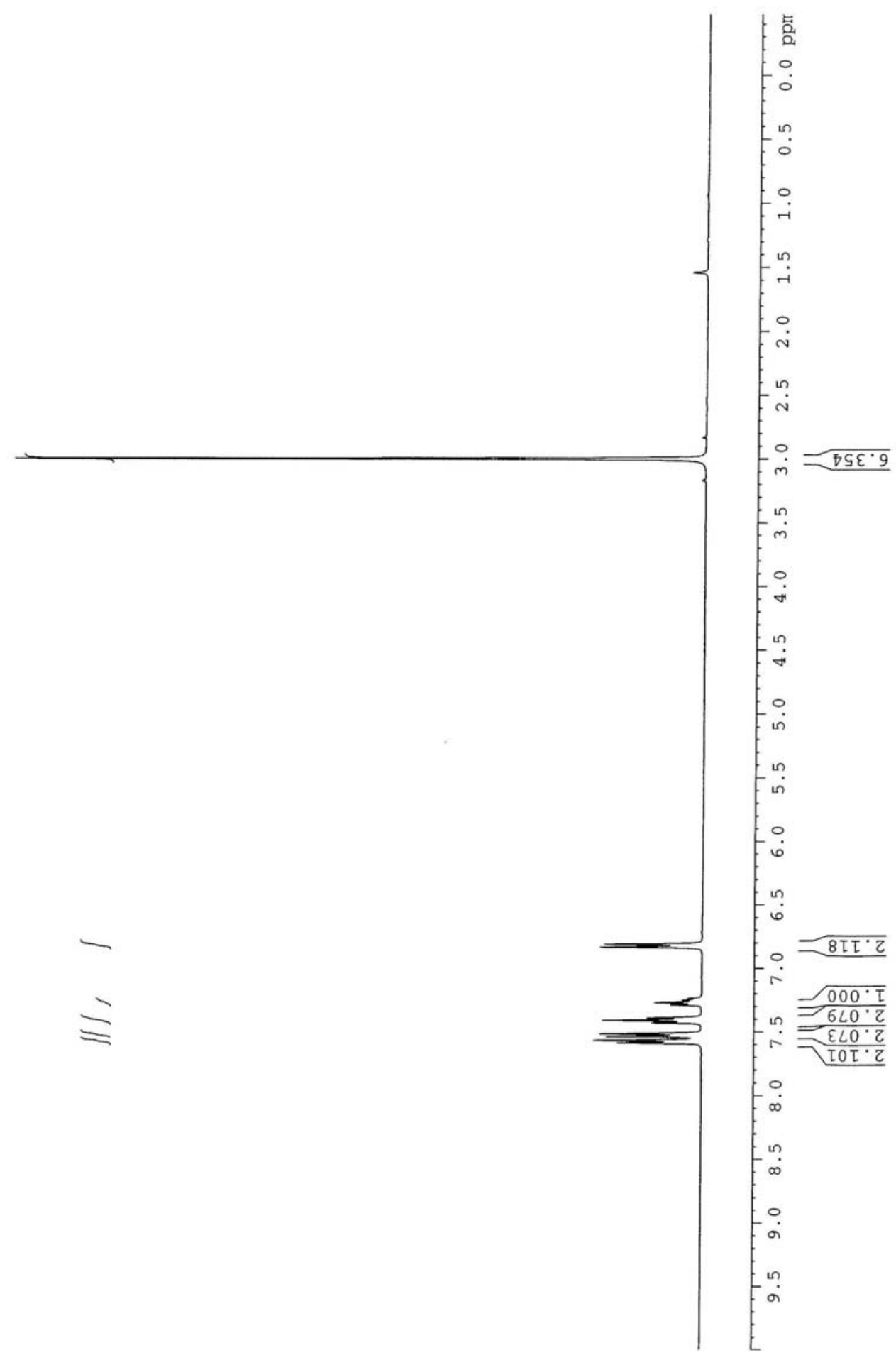


(4-Cyclohexylidenemethylphenyl)dimethylamine (Table 4, Entry 7)
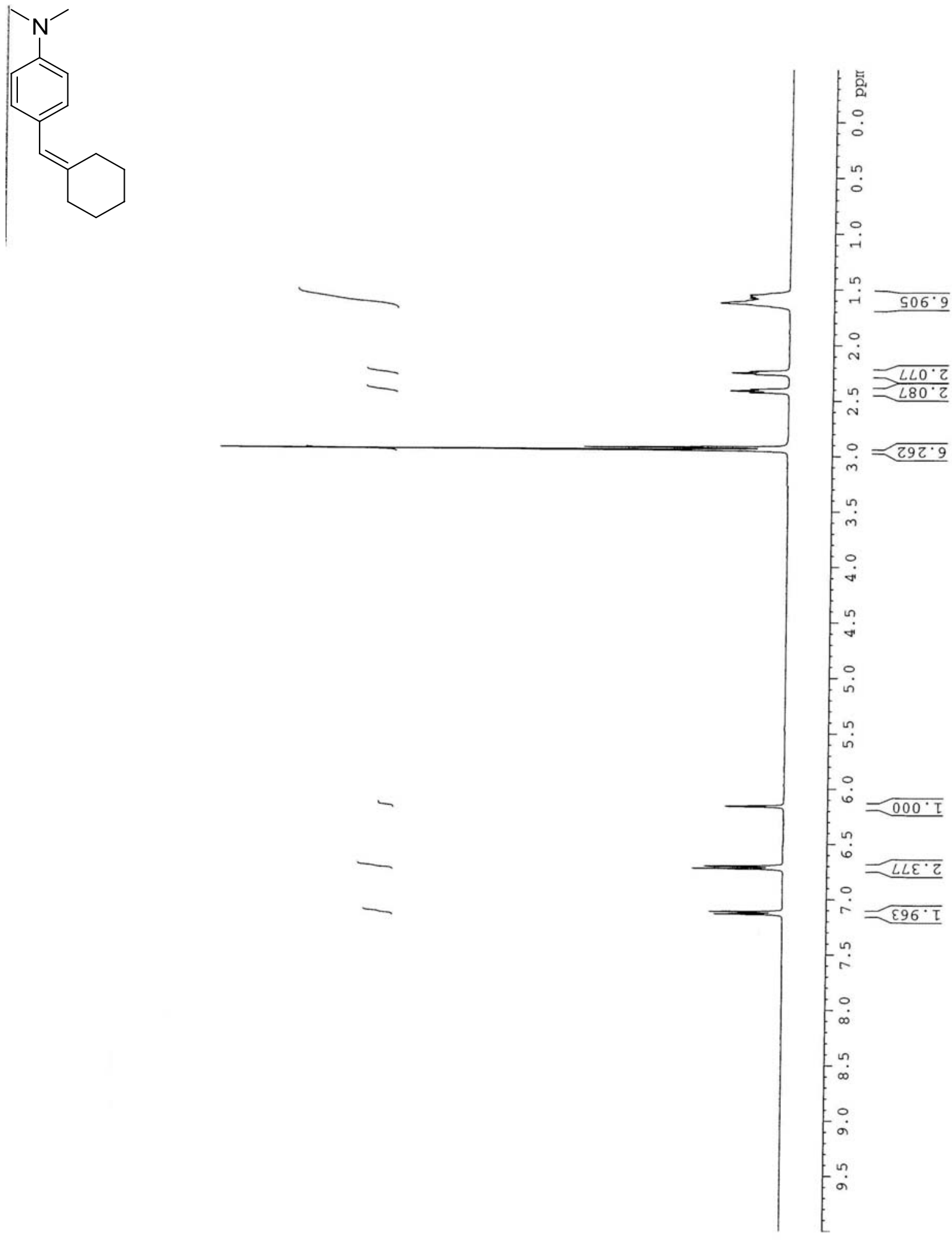
(4-Cyclohexylidenemethylphenyl)dimethylamine (Table 4, Entry 7) cont.
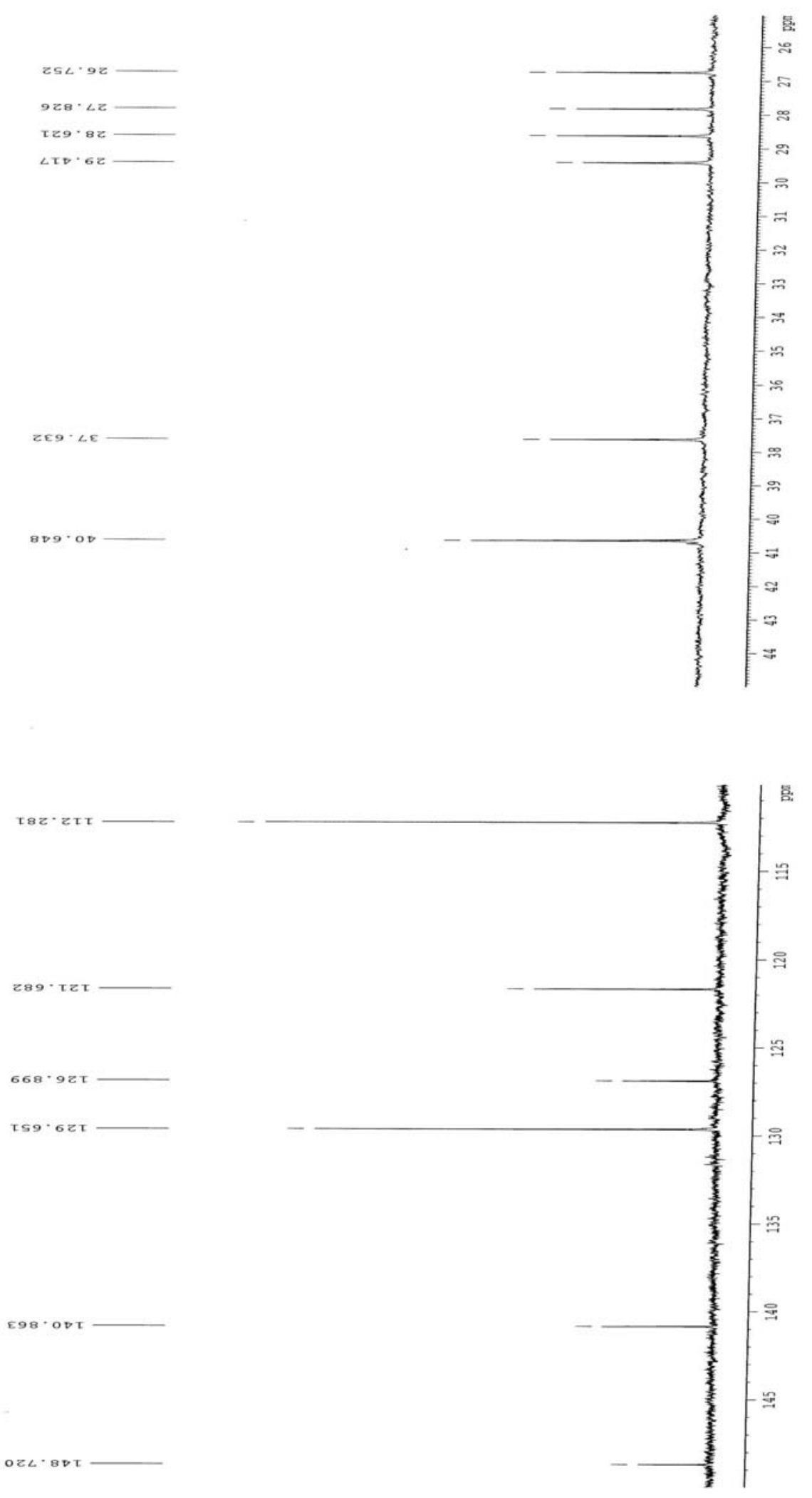


\section{(4-Cyclohexylidenemethylphenyl)dimethylamine (Table 4, Entry 7) cont.}
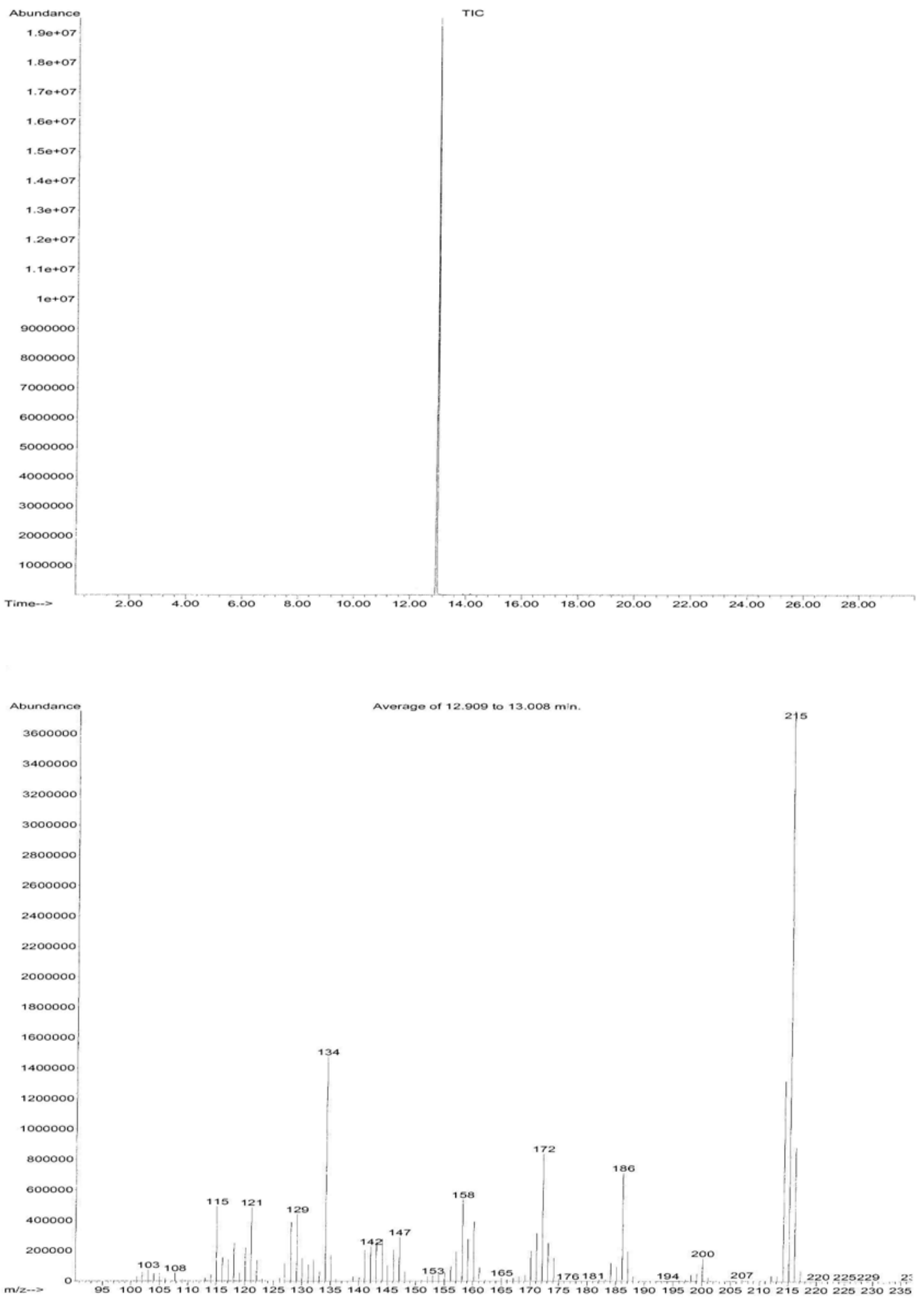
4-Trifluoromethylbiphenyl (Table 4, Entry 8)<smiles>FC(F)(F)c1ccc(-c2ccccc2)cc1</smiles>

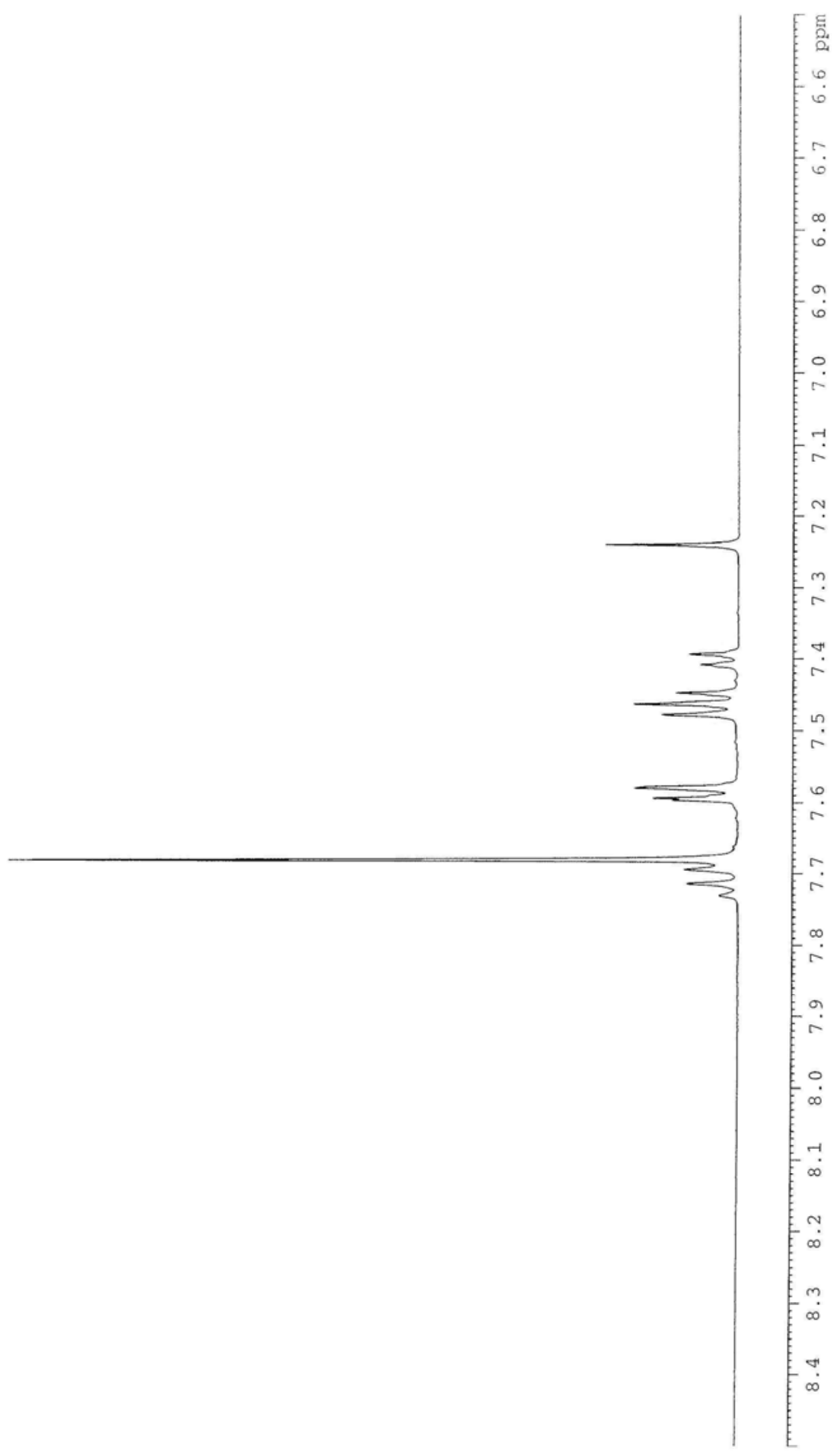


1-(4-Cyclohexylidenemethylphenoxy)-2-phenoxyethane (Table 4, Entry 9)
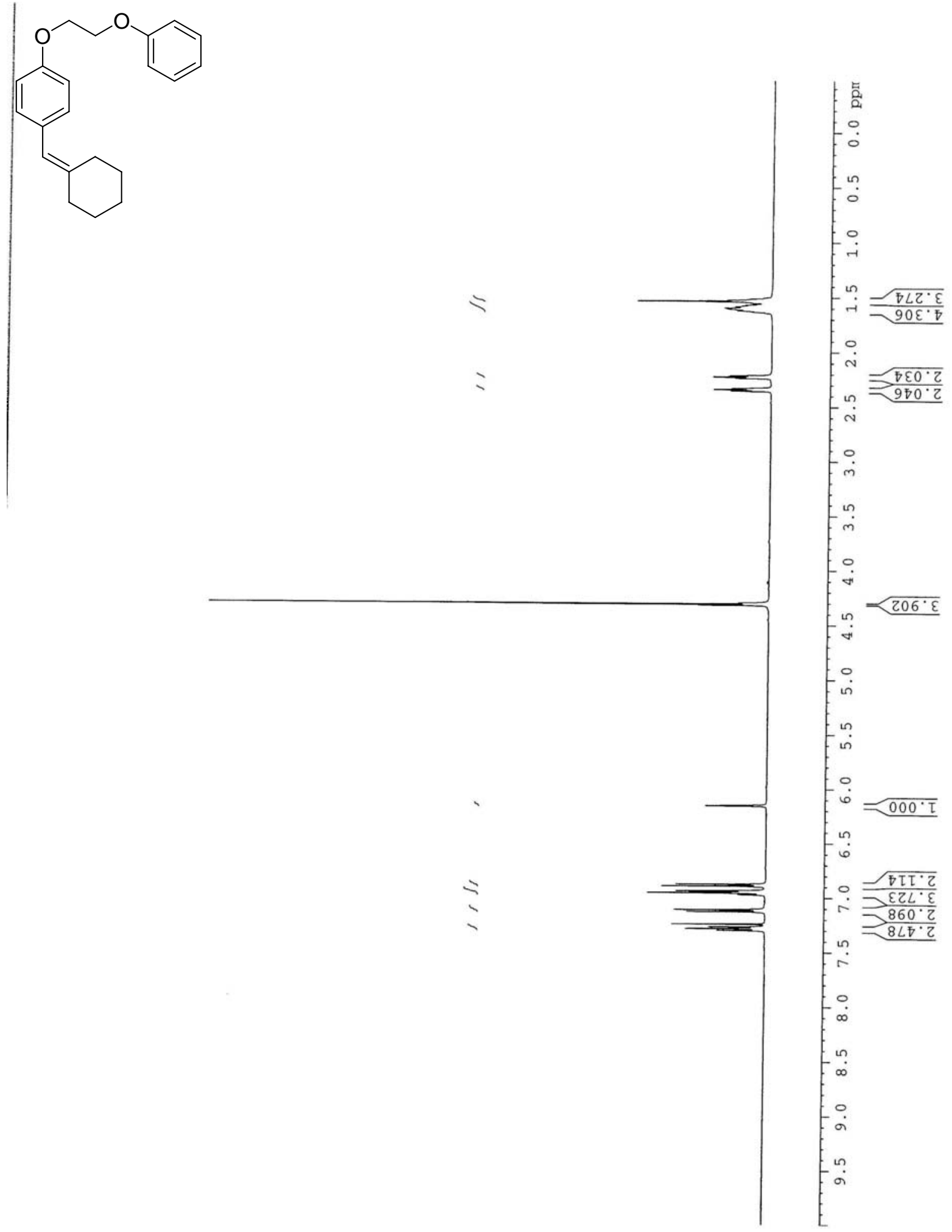
1-(4-Cyclohexylidenemethylphenoxy)-2-phenoxyethane (Table 4, Entry 9) cont.
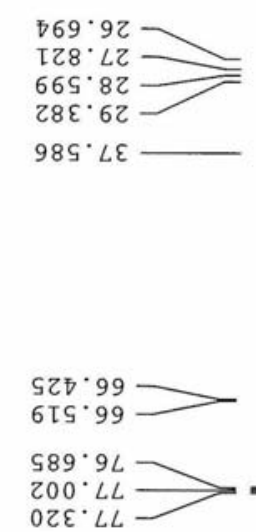

ZSZ・五T

$8 \angle 9^{\circ}$ DIT

$\angle S^{\circ}$ โZT $\longrightarrow$
$9 \mathrm{SZ}^{\circ}$ โZT

$\nabla L \nabla^{\circ} 62 T$

$266^{\circ} 62 \mathrm{\longrightarrow} \longrightarrow$

$06 \varepsilon^{\circ} \tau \varepsilon \tau$

$\nabla 8 \varepsilon^{\circ} \mathrm{\nabla \top}$

SLL. 9 ST

LI9.8ST

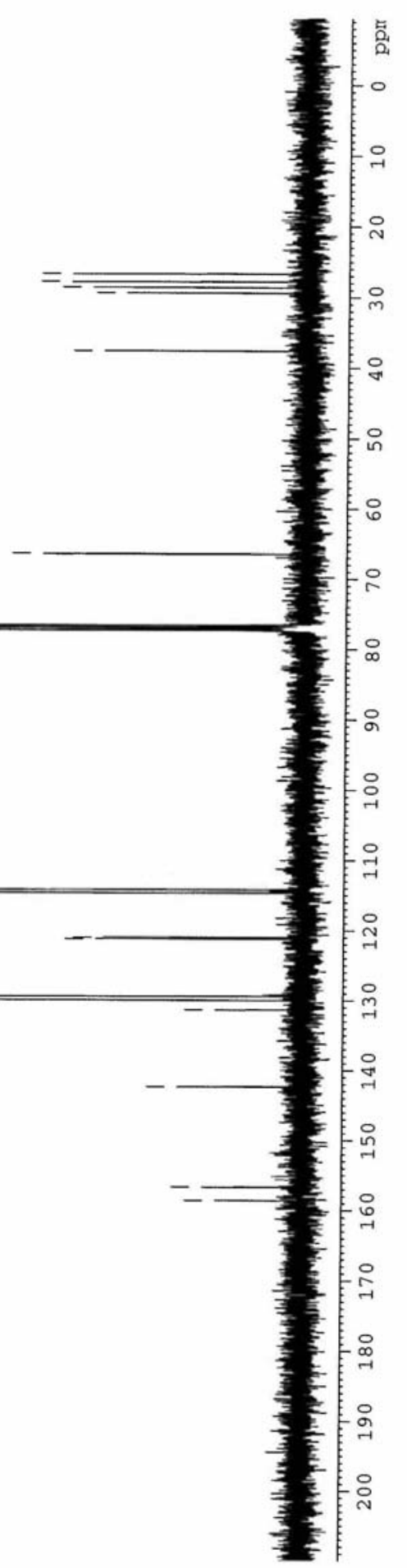


1-(4-Cyclohexylidenemethylphenoxy)-2-phenoxyethane (Table 4, Entry 9) cont.
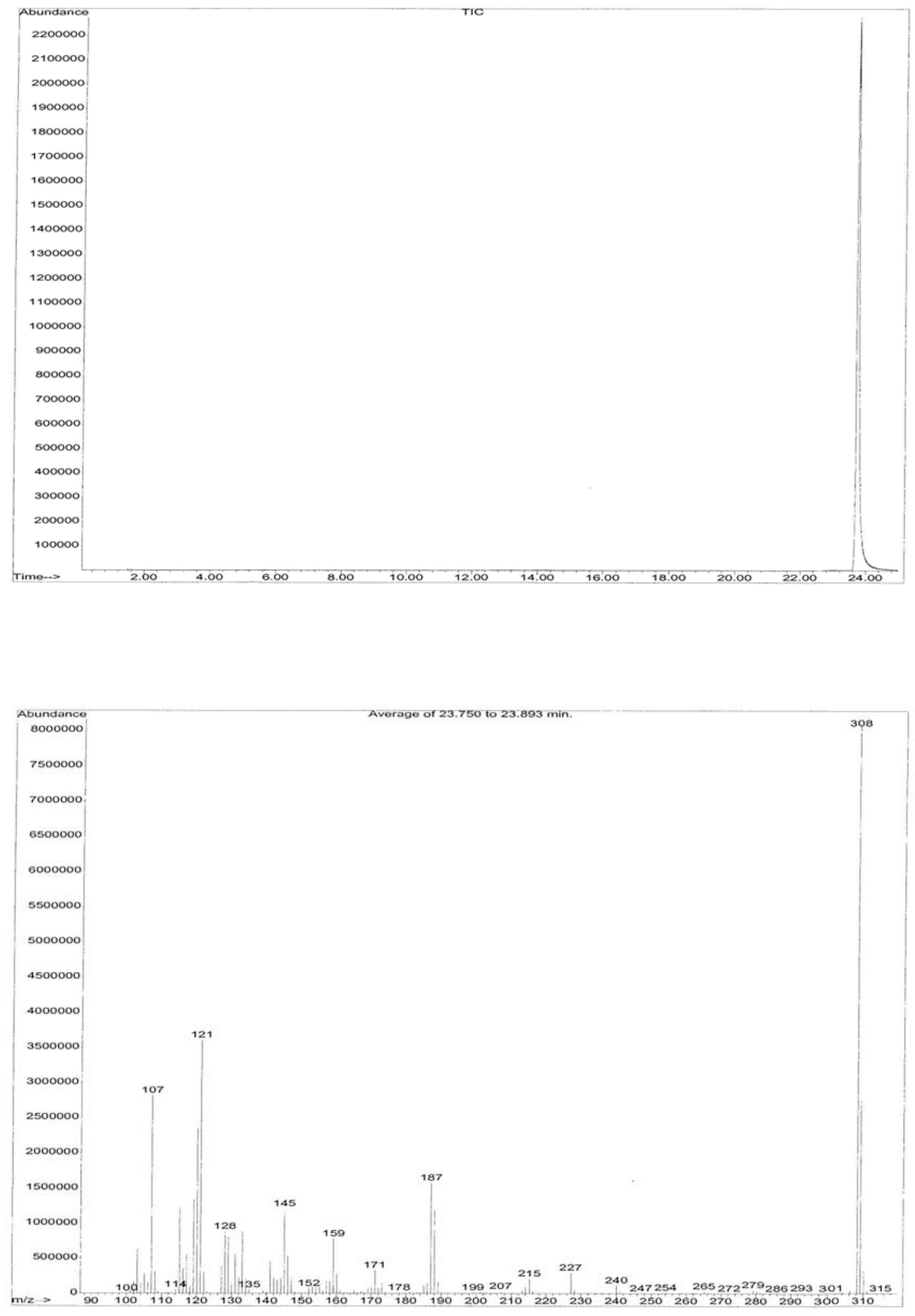